TRANSACTIONS OF THE

AMERICAN MATHEMATICAL SOCIETY

Volume 357, Number 5 , Pages 1829-1858

S 0002-9947(04)03472-5

Article electronically published on April 27, 2004

\title{
THE BASELEAF PRESERVING MAPPING CLASS GROUP OF THE UNIVERSAL HYPERBOLIC SOLENOID
}

\author{
CHRIS ODDEN \\ Dedicated to the memory of Subhashis Nag
}

\begin{abstract}
Given a closed surface $X$, the covering solenoid $\mathbf{X}_{\infty}$ is by definition the inverse limit of all finite covering surfaces over $X$. If the genus of $X$ is greater than one, then there is only one homeomorphism type of covering solenoid; it is called the universal hyperbolic solenoid. In this paper we describe the topology of $\Gamma\left(\mathbf{X}_{\infty}\right)$, the mapping class group of the universal hyperbolic solenoid. Central to this description is the notion of a virtual automorphism group. The main result is that there is a natural isomorphism of the baseleaf preserving mapping class group of $\mathbf{X}_{\infty}$ onto the virtual automorphism group of $\pi_{1}(X, *)$. This may be regarded as a genus independent generalization of the theorem of Dehn, Nielsen, Baer, and Epstein that the pointed mapping class group $\Gamma(X, *)$ is isomorphic to the automorphism group of $\pi_{1}(X, *)$.
\end{abstract}

\section{INTRODUCTION}

An $n$-solenoid is a topological space locally homeomorphic to the product of an $n$-dimensional ball and a Cantor set. Dennis Sullivan initiated the study of the Teichmüller theory of solenoids with a view towards understanding "universal" properties of dynamical systems 21]. The universal hyperbolic solenoid, denoted $\mathbf{X}_{\infty}$, is the inverse limit of a tower of finite coverings over a compact surface $X$ of genus greater than one. This space is a principal $\widehat{\pi_{1}}(X)$-bundle over $X$, where $\widehat{\pi_{1}}(X)$ is the profinite completion of $\pi_{1}(X)$.

The Teichmüller space $T\left(\mathbf{X}_{\infty}\right)$ is an object of great interest; it has been studied extensively by Sullivan, Subhashis Nag, Indranil Biswas, and Mihan Mitra ([7, [18, [3], 4], [5], [2]). A natural modular group associated to $T\left(\mathbf{X}_{\infty}\right)$ was introduced in [7] and proved to be isomorphic to the virtual automorphism group of $\pi_{1}(X)$, denoted Vaut $\left(\pi_{1}(X)\right)$. A virtual automorphism of a group is, up to easily defined equivalence, an isomorphism of finite index subgroups. In view of these facts one expects $\operatorname{Vaut}\left(\pi_{1}(X)\right)$ to be a mapping class group of some sort. This is indeed the case.

Theorem. There is a canonical isomorphism

$$
\Gamma_{B L P}\left(\mathbf{X}_{\infty}\right) \stackrel{\approx}{\longrightarrow} \operatorname{Vaut}\left(\pi_{1}(X)\right)
$$

where $\Gamma_{B L P}\left(\mathbf{X}_{\infty}\right)$ is the baseleaf preserving mapping class group of $\mathbf{X}_{\infty}$.

Received by the editors December 4, 2000 and, in revised form, July 31, 2003.

2000 Mathematics Subject Classification. Primary 57M60, 20F38; Secondary 30F60.

Key words and phrases. Virtual automorphism, mapping class group, Teichmüller theory.

(C)2004 American Mathematical Society 
This theorem is the main result of the article and appears in Section 4. It is a natural generalization of a theorem that can be traced back to the work of Dehn, Nielsen, Baer, and Epstein - namely, that there is a canonical isomorphism

$$
\Gamma(X, *) \stackrel{\approx}{\longrightarrow} \operatorname{Aut}\left(\pi_{1}(X)\right),
$$

where $\Gamma(X, *)$ denotes the basepoint preserving mapping class group of $X$.

The article is organized as follows. Section 2 consists of a general discussion of virtual automorphisms and a detailed investigation of $\operatorname{Vaut}\left(\pi_{1}(X)\right)$. This section also includes a brief review of relevant properties of profinite completions and establishes the notion of equivariant constructions. The universal hyperbolic solenoid $\mathbf{X}_{\infty}$ is the focus of Section 3. The key result here is the existence of a canonical model

$$
\mathbf{X}_{\infty} \approx \widehat{\pi_{1}}(X) \times_{\pi_{1}(X)} \tilde{X}
$$

Also of importance is an analysis of $G$-tagged solenoids, which are defined to be

$$
\mathbf{H}_{\infty}^{G}=\widehat{G} \times{ }_{G} \mathbf{D},
$$

where $G$ is a cocompact Fuchsian group and $\mathbf{D}$ is the hyperbolic plane. A $G$ tagged solenoid inherits from $G$ geometric and complex analytic structures which can be used to analyze the mapping class group of $\mathbf{H}_{\infty}^{G}$. Section 4 contains the main theorem mentioned above. This is proved first for $G$-solenoids, then in the topological setting. Another theorem establishes the existence of a homeomorphism

$$
\Gamma\left(\mathbf{X}_{\infty}\right) \approx \widehat{\pi_{1}}(X) \times_{\pi_{1}(X)} \operatorname{Vaut}\left(\pi_{1}(X)\right) .
$$

This result characterizes the topology - but not the algebraic structure - of the full mapping class group. Section 4 also contains the construction of a faithful action of $\Gamma_{B L P}\left(\mathbf{X}_{\infty}\right)$ on the unit tangent bundle of the universal hyperbolic solenoid, as well as some remarks concerning the universal euclidean solenoid.

Most results in this article appeared in the author's doctoral thesis 20]. It is a pleasure to thank my advisor John Harer for generous support and for introducing me to virtual automorphism groups. I would also like to thank Dick Hain for many stimulating conversations, as well as the referee for many helpful comments and suggestions. The paper 21] by Dennis Sullivan was an influential source.

This article is dedicated to the memory of Subhashis Nag. I am indebted to Professor Nag for his interest, encouragement, and helpful comments. His kindness and insight will be missed.

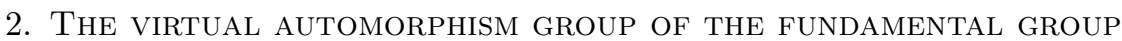 OF A CLOSED SURFACE}

In this section we define $\operatorname{Vaut}(G)$, the group of virtual automorphisms of a group $G$, and prove certain results when $G$ is the fundamental group of a closed surface. Virtual automorphism groups are also known as abstract commensurator groups or as groups of similitudes.

2.1. Virtual automorphism groups. A partial automorphism of a group $G$ is an isomorphism between two (possibly identical) subgroups of finite index in $G$. Two partial automorphisms are said to be equivalent if they agree on some finite index subgroup of the intersection of their domains. It is easy to check that this relation is in fact an equivalence. A virtual automorphism is by definition an equivalence 
class of partial automorphisms. It is straightforward to verify that $\operatorname{Vaut}(G)$, the collection of virtual automorphisms of $G$, forms a group under composition.

Example 2.1. If $G$ is a finite group, then $\operatorname{Vaut}(G)$ is trivial.

Example 2.2. The virtual automorphism group of the integers $\mathbf{Z}$ is the multiplicative group $\mathbf{Q}^{*}$ of nonzero rational numbers.

Example 2.3. Generalizing the previous example, $\operatorname{Vaut}\left(\mathbf{Z}^{n}\right) \cong G L(n, \mathbf{Q})$.

Example 2.4. The integral Heisenberg group, denoted $H_{\mathbf{Z}}$, is defined by

$$
H_{\mathbf{Z}}=\left\{\left(\begin{array}{ccc}
1 & a & b \\
0 & 1 & c \\
0 & 0 & 1
\end{array}\right) \quad: \quad a, b, c \in \mathbf{Z}\right\} .
$$

The virtual automorphism group of $H_{\mathbf{Z}}$ is the semidirect product

$$
\operatorname{Vaut}\left(H_{\mathbf{Z}}\right) \cong \mathbf{Q}^{2} \rtimes G L(2, \mathbf{Q}) .
$$

Example 2.5. Menegazzo and Tomkinson [15] constructed two examples of infinite groups with trivial virtual automorphism groups. They also proved that if $G$ is infinite and $\operatorname{Vaut}(G)$ is trivial, then $G$ is virtually a 2-group with finite central derived subgroup $[G, G]$ and $G /[G, G]$ is elementary abelian. Neither of their examples is countable; the existence of a countable group, all of whose virtual automorphisms are trivial, is an open question.

There is a connection between virtual automorphism groups and commensurators, whose definition we now recall. Let $H$ be a group and $K$ a subgroup. The commensurator of $K$ in $H$, denoted $\operatorname{Comm}_{H} K$, is

$$
\left\{\gamma \in H: \gamma K \gamma^{-1} \cap K \text { has finite index in both } K \text { and } \gamma K \gamma^{-1}\right\} \text {. }
$$

It is easily verified that $\operatorname{Comm}_{H} K$ is a subgroup of $H$. Suppose there is a pair of injective homomorphisms of a group $K$ into groups $G$ and $H$, where the image of $K$ in $G$ is of finite index. In this case there exists a natural homomorphism

$$
\operatorname{Comm}_{H} K \longrightarrow \operatorname{Vaut}(G) \text {. }
$$

Generally this map is neither injective nor surjective, as can be seen from the simple example $H=K=G=\mathbf{Z}^{n}$, where the induced map is the trivial homomorphism of $\mathbf{Z}^{n}$ into $G L(n, \mathbf{Q})$.

If $G$ can be realized as a lattice in a Lie group, then it may be possible to exploit rigidity properties of $G$ to analyze $\operatorname{Vaut}(G)$. Recall that a lattice $L$ in a Lie group $\mathcal{G}$ is a discrete subgroup of finite covolume. We will say that a Lie group $\mathcal{G}$ is Mostow rigid if every isomorphism of lattices within $\mathcal{G}$ extends uniquely to an automorphism of $\mathcal{G}$.

Proposition 2.6. If $\mathcal{G}$ is Mostow rigid and $G$ is a lattice, then there is a natural injective homomorphism

$$
\operatorname{Vaut}(G) \longrightarrow \operatorname{Aut}(\mathcal{G})
$$

Proof. In this setting a partial automorphism of $G$ is an isomorphism of lattices in $\mathcal{G}$ and therefore induces an automorphism of $\mathcal{G}$. Equivalent partial automorphisms induce the same automorphism of $\mathcal{G}$ by rigidity. Injectivity is obvious.

Proposition [2.6] is reflected in Examples 2.3] and [2.4. In both cases the virtual automorphism group of a lattice is naturally identified with the rational points in the automorphism group of the ambient Lie group. 
2.2. Virtual automorphisms of fundamental groups of surfaces. Our aim is to study the virtual automorphism group of the fundamental group of a closed surface. We shall make the following conventions. Given a surface $X$ with basepoint $*$ the fundamental group $\pi_{1}(X, *)$ will be written simply $\pi_{1}(X)$ unless special emphasis on the basepoint is required. The canonical universal cover of $X$-which is modeled on path classes in $X$ based at $*$-will be denoted $\widetilde{X}$. For every subgroup $H$ in $\pi_{1}(X)$ let $X_{H}$ denote the quotient surface $H \backslash \widetilde{X}$. We will identify a "model" for the fundamental group of a surface of genus $g$ by defining

$$
\pi_{1}(g) \stackrel{\text { def }}{=}\left\langle A_{1}, \ldots, A_{g}, B_{1}, \ldots, B_{g}: \prod_{i=1}^{g}\left[A_{i}, B_{i}\right]=1\right\rangle .
$$

There is a "grand trichotomy" in the world of Riemann surfaces based on the three universal covering surfaces: the complex plane $\mathbf{C}$, the Riemann sphere $\mathbf{P}^{1} \mathbf{C}$, and the unit disk D. This trichotomy also appears in the study of the groups $\operatorname{Vaut}\left(\pi_{1}(g)\right)$. By Examples 2.1 and 2.3

$$
\operatorname{Vaut}\left(\pi_{1}(0)\right) \cong\{1\} \text { and } \operatorname{Vaut}\left(\pi_{1}(1)\right) \cong G L(2, \mathbf{Q}) \text {. }
$$

These may be regarded as "spherical" and "euclidean" cases. There remains only the "hyperbolic case" - that is, the isomorphism class of $\operatorname{Vaut}\left(\pi_{1}(g)\right)$ stabilizes when $g>1$. This is a consequence of the following property of general virtual automorphism groups.

Proposition 2.7. Let $H_{1}$ (resp. $H_{2}$ ) be a subgroup of finite index in an arbitrary group $G_{1}$ (resp. $G_{2}$ ). An isomorphism of $H_{1}$ onto $H_{2}$ induces an isomorphism of $\operatorname{Vaut}\left(G_{1}\right)$ onto $\operatorname{Vaut}\left(G_{2}\right)$.

Proof. Let $\phi$ be an isomorphism of $H_{1}$ onto $H_{2}$ and let $\psi$ be a partial automorphism of $G_{1}$. Restricting domains if necessary, the map $\phi \circ \psi \circ \phi^{-1}$ is a partial automorphism of $G_{2}$. It is easily verified that the assignment $\psi \mapsto \phi \circ \psi \circ \phi^{-1}$ induces an isomorphism of $\operatorname{Vaut}\left(G_{1}\right)$ onto $\operatorname{Vaut}\left(G_{2}\right)$.

Corollary 2.8. If $g>1$ and $h>1$, then $\operatorname{Vaut}\left(\pi_{1}(g)\right) \cong \operatorname{Vaut}\left(\pi_{1}(h)\right)$.

Proof. A basic fact of surface topology is that if $g>1$, then there is an injective homomorphism of $\pi_{1}(g)$ into $\pi_{1}(2)$ whose image is of finite index in $\pi_{1}(2)$. It follows that the virtual automorphism groups in question are both isomorphic to $\operatorname{Vaut}\left(\pi_{1}(2)\right)$.

Remark. A stronger statement can be made. A Fuchsian group is by definition a discrete subgroup of the Lie group $\operatorname{Möb}(\mathbf{D})$ of Möbius transformations of the unit disk. By a theorem of Fox 12] every cocompact, finitely generated Fuchsian group $F$ contains a subgroup of finite index that is isomorphic to $\pi_{1}(g)$ for some $g$ greater than one. It follows that $\operatorname{Vaut}(F)$ is isomorphic to $\operatorname{Vaut}\left(\pi_{1}(2)\right)$.

A virtual automorphism of the fundamental group of a surface encodes certain topological data. Fix a closed, pointed surface $(X, *)$ and let $\psi: A \rightarrow B$ be a partial automorphism of $\pi_{1}(X)$. The subgroups $A$ and $B$ correspond to unique pointed covering surfaces $\left(X_{A}, *\right)$ and $\left(X_{B}, *\right)$. To $\psi$ there corresponds a pointed homotopy class of basepoint respecting homeomorphism of $X_{A}$ onto $X_{B}$. The partial automorphism $\psi$ thus yields the not necessarily commutative diagram shown in Figure 1. By restricting the domain of $\psi$ it is possible to pass to a higher cover in the diagram. This freedom allows for the composition of two such diagrams as in 


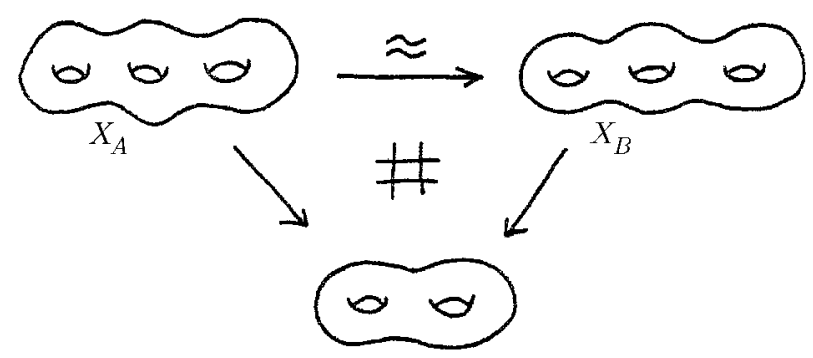

Figure 1. Topological data of a partial automorphism.

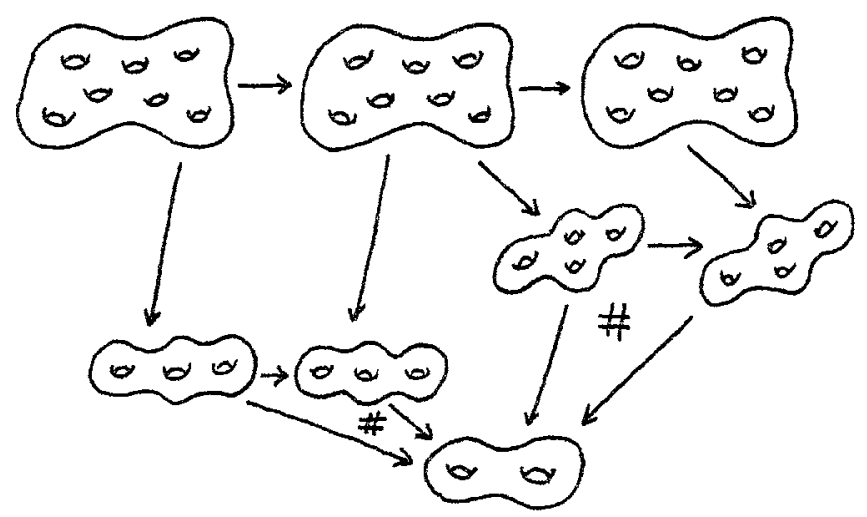

FIGURE 2. Composing partial automorphisms.

Figure 2. It follows that a virtual automorphism may be viewed as an equivalence class of such noncommuting diagrams, where equivalence subsumes two relations: pointed homotopy equivalence and the equivalence introduced by lifting to higher covers.

Let $\phi$ and $\psi$ be partial automorphisms of $G$ defined respectively on subgroups $A$ and $B$. If $\phi$ and $\psi$ agree on $A \cap B$, then $\phi$ is equivalent to $\psi$; however, the converse need not hold - indeed, $\phi$ and $\psi$ may agree only on a subgroup of "very high" index in $A \cap B$. This makes the general problem of recognizing equivalent partial automorphisms difficult. Happily there is a class of groups - which contains the groups $\pi_{1}(g)$ - for which a necessary and sufficient condition for equivalence is agreement on the common domain. Before turning to this class of groups we prove the following lemma.

Lemma 2.9. Let $G$ be an arbitrary group and let $H$ be a subgroup of index $n$. If $\gamma \in G$, then $\gamma^{n !} \in H$.

Proof. Left multiplication induces a homomorphism of $G$ into the group of permutations of the left cosets of $H$ in $G$. Since the latter group has order $n$ ! it follows that the image of $\gamma^{n !}$ is trivial, which implies $\gamma^{n !}$ is an element of $H$.

An $R$-group is defined by the following property: every equation of the form $x^{n}=a$ has no more than one solution in $x$. In other words, the extraction of roots in an $R$-group is unique [13]. If $X$ is a closed surface, then $\pi_{1}(X)$ is an $R$-group. 
Certainly this is the case for genus one; that is, when $\pi_{1}(X)=\mathbf{Z}^{2}$. For $X$ of genus greater than one, choose an isomorphism of $\pi_{1}(X)$ onto a Fuchsian group $G$. Every element of $G$ is a translation along some geodesic axis. It is well known that two elements of $G$ commute if and only if their axes coincide; therefore, if $x^{n}=\gamma$ and $\gamma$ is a translation of length $l$ along the axis $A$, then $x$ is a translation of length $l / n$ along $A$. It follows that $\pi_{1}(X)$ is an $R$-group. (Other examples of $R$-groups are free groups and torsion free locally nilpotent groups [14.)

Proposition 2.10. Let $G$ be an R-group. If two partial automorphisms of $G$ are equivalent, then they agree on the intersection of their domains.

Proof. Suppose $\phi$ and $\psi$ are equivalent partial automorphisms of $G$. Let $K$ be the intersection of their domains. By hypothesis $K$ contains a subgroup $A$, of some finite index $n$ in $K$, on which $\phi$ and $\psi$ agree. Lemma 2.9 implies that if $\gamma \in K$, then $\gamma^{n !} \in A$; therefore,

$$
\phi\left(\gamma^{n !}\right)=\psi\left(\gamma^{n !}\right) .
$$

Since $\gamma$ is in the common domain of $\phi$ and $\psi$,

$$
\phi(\gamma)^{n !}=\psi(\gamma)^{n !},
$$

and since $G$ is an $R$-group, $\phi(\gamma)=\psi(\gamma)$.

Equivalence of partial automorphisms is thus a rigid relation for $R$-groups. This rigidity implies the following "unique continuation" property.

Proposition 2.11. Let $\phi$ be a partial automorphism of an $R$-group $G$, let $H$ be the domain of $\phi$, and let $K$ be a subgroup that contains $H$. If there exists an extension of $\phi$ to a partial automorphism defined on $K$, then this extension is unique.

Proof. Any two such extensions are necessarily equivalent. By Proposition 2.10 the extensions must agree on $K$.

Corollary 2.12. Let $H$ be a subgroup of finite index in an $R$-group $G$. The inclusion of $H$ in $G$ induces a natural, injective homomorphism

$$
\operatorname{Aut}(H) \longrightarrow \operatorname{Vaut}(G) \text {. }
$$

Proof. A map of $\operatorname{Aut}(H)$ into $\operatorname{Vaut}(G)$ exists since every automorphism of $H$ represents a virtual automorphism of $G$. It is easily verified that this map is a homomorphism. If $\psi$ is an automorphism of $H$ that represents the trivial virtual automorphism of $G$, then $\psi$ is equivalent to the identity automorphism on some subgroup of finite index in $H$. By Proposition $2.11 \psi$ must be the trivial automorphism of $H$.

Corollary 2.13. If $g$ and $h$ are both integers greater than one, then there is an injective homomorphism

$$
\operatorname{Aut}\left(\pi_{1}(g)\right) \longrightarrow \operatorname{Vaut}\left(\pi_{1}(h)\right) .
$$

Proof. By Corollary 2.8 we may assume $h=2$. As noted in the proof of that corollary, $\pi_{1}(g)$ can be embedded in $\pi_{1}(2)$ as a subgroup of finite index.

Let $(X, *)$ be a pointed, closed surface. By definition the pointed mapping class group of $X$, denoted $\Gamma(X, *)$, is the group of pointed isotopy classes of basepoint preserving self-homeomorphisms of $X$. The following theorem can be traced back to the work of Max Dehn, Jacob Nielsen, Reinhold Baer, and D. A. Epstein. 
Theorem 2.14 (Dehn, Nielsen, Baer, Epstein). There is a natural isomorphism

$$
\Gamma(X, *) \stackrel{\cong}{\longrightarrow} \operatorname{Aut}\left(\pi_{1}(X, *)\right) .
$$

Proof. By the Dehn-Neilsen Theorem [19] every automorphism is induced by a mapping class. Epstein [10, extending the work of Baer [1], proved that if a homeomorphism induces the identity automorphism of $\pi_{1}(X)$, then it is isotopicvia a basepoint preserving isotopy - to the identity map on $X$.

Suppose $(Y, *)$ is a finite covering surface of $(X, *)$. By combining the DehnNielsen-Baer-Epstein theorem and Corollary 2.12 one obtains an injective map

$$
\Gamma(Y, *) \longrightarrow \operatorname{Vaut}\left(\pi_{1}(X, *)\right) \text {. }
$$

In other words, the pointed mapping class group of every covering surface of $(X, *)$ appears as a subgroup of $\operatorname{Vaut}\left(\pi_{1}(X)\right)$. We shall have more to say about this in Section 4.3.

There are, of course, many embeddings of Aut $\left(\pi_{1}(g)\right)$ in $\operatorname{Vaut}\left(\pi_{1}(X)\right)$. One might wonder if the union of the images of all such embeddings covers Vaut $\left(\pi_{1}(X)\right)$. Put another way, is every virtual automorphism of $\pi_{1}(X)$ represented by some automorphism defined on a subgroup of finite index in $\pi_{1}(X)$ ? The following theorem answers this question negatively.

Theorem 2.15. Let $X$ be a closed surface of genus greater than one. There exists a virtual automorphism of $\pi_{1}(X)$ that cannot be represented by an automorphism of some subgroup of finite index in $\pi_{1}(X)$.

Proof. Figure 3 depicts a homeomorphism between a pair of genus three surfaces, both of which cover a surface of genus two. Both covering maps are quotient maps associated to the $\mathbf{Z} / 2$-action of rotating a surface $180^{\circ}$ about its "center hole". (The inverse image of a dashed curve is drawn in the left-hand covering surface and the inverse image of a jagged curve is drawn in the right-hand covering surface.) The homeomorphism is obtained by "permuting holes"; it can be constructed by doubling an appropriate self-homeomorphism of a thrice-punctured disk. Let $\psi$ denote the partial automorphism of $\pi_{1}(X)$ induced by this homeomorphism.

The key feature of $\psi$ is that the curve $\gamma$ is not in the domain of $\psi$ while $\psi\left(\gamma^{2}\right)=\gamma$. This property allows us to prove the proposition by contradiction. Assume that $\psi$ is equivalent to an automorphism $\theta$ defined on a subgroup $H$. By Lemma 2.9 there exists $N$ for which $\gamma^{N} \in H$. Equivalent representatives of a virtual mapping class agree on the intersection of their domains; therefore, $\theta\left(\gamma^{2 N}\right)=\gamma^{N}$. Since $\theta$ is an automorphism of $H$, the formula

$$
\theta^{k}\left(\gamma^{N}\right)=\gamma^{\left(1 / 2^{k}\right) N}
$$

holds for all $k$. But this is absurd.

Similar constructions exist when the genus of $X$ is greater than two.

The partial automorphism constructed in the preceding proof is a "square root" mapping on the curve $\gamma$. Arbitrary "rational root" mappings exist in the following sense: for every $\gamma$ in $\pi_{1}(X)$ and every pair $p, q$ of relatively prime positive integers, there exists a partial automorphism that maps $\gamma^{q}$ to $\gamma^{p}[20]$.

Define Fiat $(G)$ to be the subgroup of $\operatorname{Vaut}(G)$ generated by all automorphisms of subgroups of finite index in $G$. In [20] it was shown that if $G=\pi_{1}(g)$ and $g>2$, then Fiat $(G)$ is normal in $\operatorname{Vaut}(G)$ and the isomorphism class of Fiat $(G)$ is 


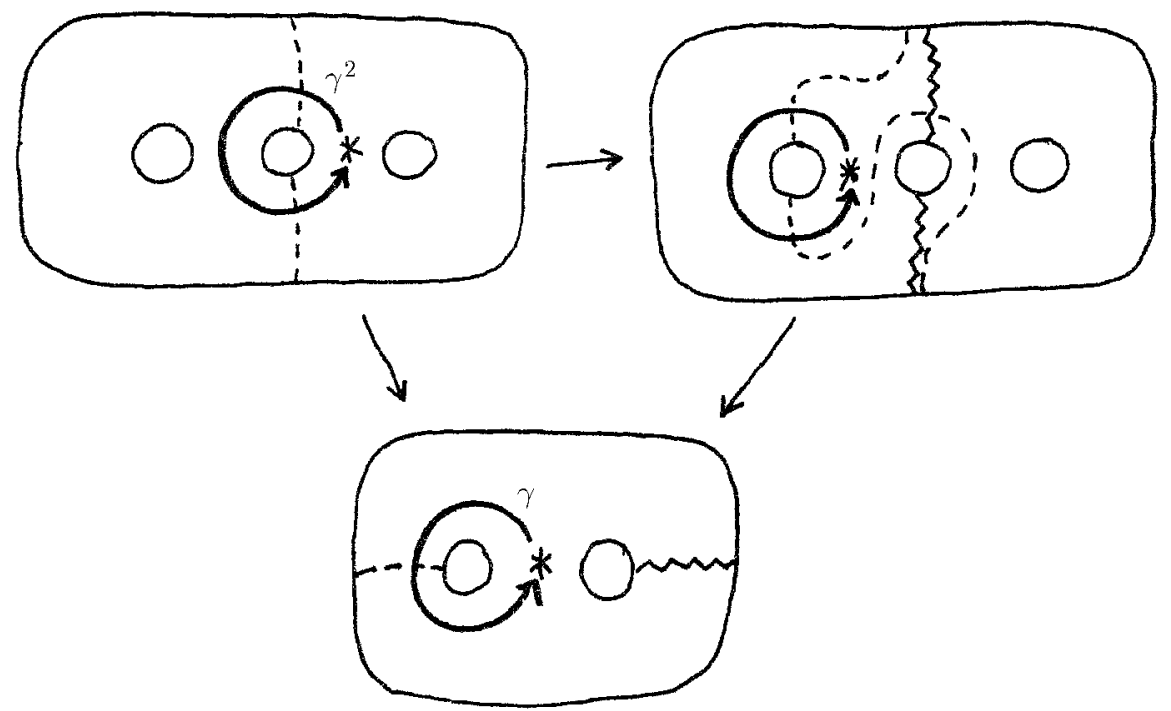

FiguRE 3. A virtual automorphism that does not arise from an automorphism.

independent of $g$. An open question: what is $\operatorname{Vaut}(G) / \operatorname{Fiat}(G)$ ? In particular, is this group trivial?

Remark. The notation Fiat is meant to suggest finite index automorphism. In [20] each generator of $\operatorname{Fiat}(G)$ is called mapping class like since it corresponds to a pointed mapping class of some covering surface. It should be noted that every virtual automorphism - mapping class like or not - arises from a mapping class of the universal hyperbolic solenoid; see Theorem 4.10

2.3. Profinite completions. The profinite completion of a group $G$ is the projective limit of the finite quotients of $G$. This section is a brief summary of the properties of profinite completions that will be used later.

Assume that $G$ is a finitely generated, residually finite group. Define $A_{n}$ to be the intersection of all subgroups of index $n$ or less in $G$. Since $G$ is finitely generated there are only a finite number of subgroups of a given index; therefore, $A_{n}$ is of finite index in $G$. It is easily verified that $A_{n}$ is normal (in fact characteristic) in $G$, and that the collection $\left\{A_{n}\right\}$ is a linearly ordered neighborhood base of the identity for the profinite topology. Since $G$ is residually finite, the profinite topology is Hausdorff.

The subgroups $A_{n}$ can be used to construct a nonarchimedean metric that generates the profinite topology of $G$. For every nontrivial $\gamma$ in $G$ define

$$
\operatorname{ord}(\gamma)=\max \left\{n: \gamma \in A_{n}\right\}
$$

and set $\operatorname{ord}(1)=\infty$. For every $\gamma$ and $\zeta$ in $G$, set

$$
\rho(\gamma, \zeta)=e^{-\operatorname{ord}\left(\gamma^{-1} \zeta\right)}
$$

The function $\rho$ is a nonarchimedean metric on $G$; moreover, the completion of $G$ with respect to $\rho$ is the profinite completion $\widehat{G}$ of $G$.

Let $\epsilon$ be a positive number and define $N(\epsilon)$ to be the smallest natural number $n$ that satisfies $e^{-n}<\epsilon$. A pleasant and useful fact is that for every positive $\epsilon$ there 
are a finite number of open $\epsilon$-balls in $\widehat{G}$, and these $\epsilon$-balls are precisely the cosets in $\widehat{G}$ by the group $\widehat{A}_{N(\epsilon)}$.

The following lemma is elementary; its proof is omitted.

Lemma 2.16. Let $H$ be a subgroup of finite index in $G$. A system of right (resp. left) coset representatives of $G$ modulo $H$ is also a system of right (resp. left) coset representatives of $\widehat{G}$ modulo $\widehat{H}$.

A consequence of this lemma is that for every $t$ in $\widehat{G}$ and every subgroup $H$ of finite index in $G$, it is possible to express $t$ as a product $h \gamma$, where $h \in \widehat{H}$ and $\gamma \in G$.

2.4. Equivariant constructions. In this section we introduce the notion of equivariance. An immediate application is the construction of a homomorphism of $\operatorname{Vaut}(G)$ into the permutation group $\operatorname{Sym}(G \backslash \widehat{G})$. Equivariant constructions will be used throughout Sections 3 and 4.

Definition 2.17. Let $G_{1}$ (resp. $G_{2}$ ) be a group that acts by homeomorphisms on the topological space $X_{1}$ (resp. $X_{2}$ ). A homeomorphism $f$ of $X_{1}$ onto $X_{2}$ is equivariant with respect to $G_{1}$ and $G_{2}$ if there exists an isomorphism $\phi_{f}$ of $G_{1}$ onto $G_{2}$ such that, for all $x$ in $X_{1}$ and for all $g$ in $G_{1}$,

$$
f(g \cdot x)=\phi_{f}(g) \cdot f(x) .
$$

When the context is clear we will simply say that $f$ is equivariant.

The primary application of equivariance is embodied in the following trivial fact: if $f$ is a homeomorphism of $X_{1}$ onto $X_{2}$ that is equivariant with respect to groups $G_{1}$ and $G_{2}$, then $f$ induces a homeomorphism between the quotient spaces $G_{1} \backslash X_{1}$ and $G_{2} \backslash X_{2}$.

Proposition 2.18. If $H$ is a subgroup of finite index in $G$, then there is a natural bijective map of $H \backslash \widehat{H}$ onto $G \backslash \widehat{G}$.

Proof. Let $i$ be the composite map

$$
\widehat{H} \hookrightarrow \widehat{G} \rightarrow G \backslash \widehat{G} .
$$

This map is surjective, for Lemma 2.16 guarantees that every $t$ in $\widehat{G}$ can be expressed as a product $\gamma s$, where $\gamma \in G$ and $s \in \widehat{H}$; hence the equivalence class $i(s)$ contains $t$.

Suppose $i\left(s_{1}\right)=i\left(s_{2}\right)$; that is, there exists some $\gamma$ in $G$ such that $\gamma s_{1}=s_{2}$. It follows that $s_{2} s_{1}^{-1}$ lies in the intersection of $G$ and $\widehat{H}$, which is just $H$. The map $i$ is therefore defined on $H \backslash \widehat{H}$ and it is trivially injective.

Let $\operatorname{Sym}(G \backslash \widehat{G})$ denote the group of permutations of the set $G \backslash \widehat{G}$. We will now construct a natural homomorphism

$$
\operatorname{Vaut}(G) \longrightarrow \operatorname{Sym}(G \backslash \widehat{G}) .
$$

Let $\phi: H \rightarrow K$ be a partial automorphism of $G$. It induces an isomorphism of $\widehat{H}$ onto $\widehat{K}$ which in turn induces a bijective map of $H \backslash \widehat{H}$ onto $K \backslash \widehat{K}$. This bijective map will also be denoted $\phi$. By Proposition 2.18]both $H \backslash \widehat{H}$ and $K \backslash \widehat{K}$ are identified 
with $G \backslash \widehat{G}$. Pulling $\phi$ back via these identifications we obtain a bijective map $\widetilde{\phi}$ of $G \backslash \widehat{G}$ onto itself:

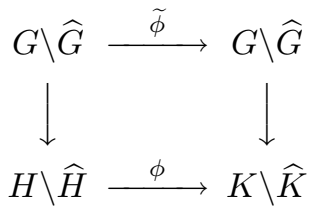

The assignment $[\phi] \mapsto \widetilde{\phi}$ defines a homomorphism of $\operatorname{Vaut}(G)$ into $\operatorname{Sym}(G \backslash \widehat{G})$.

\section{The UniVERSAL HYPERBOLIC SOLENOID}

An $n$-dimensional solenoid is a compact topological space locally homeomorphic to the product of an $n$-dimensional ball and a totally disconnected space. For example, if $C$ is the standard ternary Cantor set and $f$ is a self-homeomorphism of $C$, then the mapping cylinder $C \times_{f}[0,1]$ is a one-solenoid. An extensive list of examples, applications, and references can be found in [21.

In this section we will examine the topology of the universal hyperbolic solenoid as well as complex analytic and geometric structures which the universal hyperbolic solenoid supports.

3.1. Covering solenoids. Covering solenoids are defined by taking the inverse limit of a tower of covering surfaces over a fixed base space.

Example 3.1. The euclidean one-solenoid, denoted $\mathbf{E}_{\infty}^{1}$, is by definition the inverse limit of the system of all finite covers of the circle. A cofinal, linearly ordered subsystem of covers is

$$
\cdots \longleftarrow \mathbf{R} /(n-1) ! \mathbf{Z} \longleftarrow \mathbf{R} / n ! \mathbf{Z} \longleftarrow \mathbf{R} /(n+1) ! \mathbf{Z} \longleftarrow \cdots .
$$

The space $\mathbf{E}_{\infty}^{1}$ is compact and fibers over the circle; each fiber is homeomorphic to $\widehat{\mathbf{Z}}$, the profinite completion of the integers.

Example 3.2. The level $n$ congruence group is the kernel of the natural surjection

$$
S L(2, \mathbf{Z}) \longrightarrow S L(2, \mathbf{Z} / n \mathbf{Z}) .
$$

Let $\Gamma(n)$ denote the image of this group in $\operatorname{PSL}(2, \mathbf{Z})$. If $n \geq 2$, then $\Gamma(n)$ is torsion free and acts freely on $\mathbf{H}$. The quotient spaces $\Gamma(n) \backslash \mathbf{H}$ form an inverse system of covering spaces over the Riemann surface $S=P S L(2, \mathbf{Z}) \backslash \mathbf{H}$, which has one cusp and two singular points. Away from the singular points the inverse limit

$$
\mathcal{H}_{\infty}=\lim _{\overleftarrow{n}} \Gamma(n) \backslash \mathbf{H}
$$

fibers over $S$; each fiber is homeomorphic to the profinite completion of $\operatorname{PSL}(2, \mathbf{Z})$. A thorough discussion of $\mathcal{H}_{\infty}$ can be found in [17].

Besides being an important object in its own right the euclidean one-solenoid $\mathbf{E}_{\infty}^{1}$ is the one-dimensional analogue of the object we wish to study; therefore, we take a moment to examine this space closely. If $f$ is the self-homeomorphism of $\widehat{\mathbf{Z}}$ defined by $x \mapsto x+1$, then

$$
\mathbf{E}_{\infty}^{1} \approx \widehat{\mathbf{Z}} \times_{f}[0,1]
$$


The map $f$ encodes the monodromy action of $\pi_{1}\left(S^{1}\right)$ on the fiber $\widehat{\mathbf{Z}}$. An equivalent construction of $\mathbf{E}_{\infty}^{1}$ is to take the quotient of $\widehat{\mathbf{Z}} \times \mathbf{R}$ by the diagonal action of $\mathbf{Z}$; thus,

$$
\mathbf{E}_{\infty}^{1} \approx \widehat{\mathbf{Z}} \times_{\mathbf{Z}} \mathbf{R}
$$

We mention in passing a third view of this space: $\mathbf{E}_{\infty}^{1}$ is naturally homeomorphic to $\mathbf{A} / \mathbf{Q}$, the adèles modulo the discrete action of the rational numbers [17.

Let $(X, *)$ be a pointed, closed surface. The collection of finite index subgroups of $\pi_{1}(X)$ is ordered by inclusion. The corresponding tower of canonical, pointed covering surfaces and their projections is an inverse system of topological spaces.

Definition 3.3. The canonical covering solenoid $\mathbf{X}_{\infty}$ is the inverse limit of the tower of canonical, closed covering surfaces over $X$; that is,

$$
\mathbf{X}_{\infty} \stackrel{\text { def }}{=}{\underset{H}{\lim }}_{H} X_{H}
$$

where $H$ ranges over all subgroups of finite index in $\pi_{1}(X)$.

At this point the grand trichotomy to which we alluded in Section 2.2 reappears: for closed surfaces there are only three homeomorphism classes of covering solenoids. If the genus of $X$ is zero, then $\mathbf{X}_{\infty}$ is simply the two-sphere. If $X$ is a torus, then by definition we obtain the euclidean two-solenoid $\mathbf{E}_{\infty}$. There exist homeomorphisms

$$
\mathbf{E}_{\infty} \approx \widehat{\mathbf{Z}}^{2} \times_{\mathbf{Z}^{2}} \mathbf{R}^{2} \approx(\mathbf{A} / \mathbf{Q})^{2}
$$

that are analogous to the one-dimensional case. (We will confine our remarks on $\mathbf{E}_{\infty}$ to Section 4.5)

The next proposition is a standard result; it asserts that the canonical covering solenoids over the remaining surfaces are homeomorphic. It therefore makes sense to speak of the universal hyperbolic solenoid.

Proposition 3.4. If $X$ and $Y$ are closed surfaces, both of genus greater than one, then $\mathbf{X}_{\infty}$ is homeomorphic to $\mathbf{Y}_{\infty}$.

Proof. The groups $\pi_{1}(X)$ and $\pi_{1}(Y)$ contain subgroups of finite index that are isomorphic; consequently, the inverse systems of covering surfaces contain homeomorphic, cofinal subsystems. It follows that $\mathbf{X}_{\infty}$ is homeomorphic to $\mathbf{Y}_{\infty}$.

A path-connected component of $\mathbf{X}_{\infty}$ is a leaf. Given a closed curve in $X$ there exists a finite covering surface of $X$ in which the lift of the curve fails to close. (This reflects the fact that $\pi_{1}(X)$ is residually finite.) It follows that every leaf of $\mathbf{X}_{\infty}$ is simply connected.

In order to study the algebraic topology of a surface it is often necessary to recognize some distinguished feature on the surface - a basepoint, for instance. On a solenoid another option is to distinguish a baseleaf. A third possibility is a marked baseleaf - by definition a space $l$ and a continuous, bijective map of $l$ onto a leaf of the solenoid. Marked baseleafs are fundamental to our study of the universal hyperbolic solenoid.

The covering solenoid $\mathbf{X}_{\infty}$ carries both a canonical basepoint and a canonical marked baseleaf. The canonical basepoint is determined by the collection of basepoints in the covering tower $\left\{X_{H}\right\}$. The marked baseleaf is modeled on the universal cover $\widetilde{X}$ : the collection of projections $\left\{\widetilde{X} \rightarrow X_{H}\right\}$ is compatible with the inverse 
system of covering maps over $X$; by the universal mapping property of $\mathbf{X}_{\infty}$ this collection defines a continuous map of $\widetilde{X}$ into $\mathbf{X}_{\infty}$.

The fundamental group $\pi_{1}(X)$ acts on $\widetilde{X}$ by homeomorphisms; $\pi_{1}(X)$ also acts on itself - and therefore on its profinite completion $\widehat{\pi_{1}}(X)$-by right translation. Define an action of $\pi_{1}(X)$ on the product $\widehat{\pi_{1}}(X) \times \widetilde{X}$ by the formula

$$
\gamma \cdot(t, x) \stackrel{\text { def }}{=}\left(t \gamma^{-1}, \gamma x\right) \text {. }
$$

The quotient space $\widehat{\pi_{1}}(X) \times_{\pi_{1}(X)} \tilde{X}$ is a compact, principal $\widehat{\pi_{1}}(X)$-bundle over $X$. This space will serve as the canonical model of $\mathbf{X}_{\infty}$.

Theorem 3.5. There is a canonical homeomorphism

$$
\widehat{\pi_{1}}(X) \times_{\pi_{1}(X)} \tilde{X} \stackrel{\approx}{\longrightarrow} \mathbf{X}_{\infty} .
$$

Proof. To ease the burden of notation write $G=\pi_{1}(X)$. Since $\widehat{G} \times_{G} \widetilde{X}$ is compact and $\mathbf{X}_{\infty}$ is Hausdorff it is enough to exhibit a continuous bijection between these spaces.

For every subgroup $H$ of finite index in $G$, define the map

$$
\widehat{G} \times \widetilde{X} \stackrel{f_{H}}{\longrightarrow} X_{H}
$$

in the following manner. Let $(t, x)$ be in $\widehat{G} \times \widetilde{X}$. There exists $\gamma$ in $G$ such that $t \gamma^{-1} \in \widehat{H}$; hence $\left(t \gamma^{-1}, \gamma x\right) \in \widehat{H} \times \widetilde{X}$. Set $f_{H}(t, x)$ equal to the projection of $\gamma x$ onto the surface $X_{H}$.

We must check that the definition of $f_{H}$ does not depend on the choice of $\gamma$. Suppose $\zeta$ is another element of $G$ such that $t \zeta^{-1} \in \widehat{H}$. An easy calculation shows that $\gamma \zeta^{-1} \in \widehat{H}$ and it is trivially true that $\gamma \zeta^{-1} \in G$; therefore $\gamma \zeta^{-1} \in H$. It follows that $\gamma x$ and $\zeta x$ are projected onto the same point in $X_{H}$.

An argument with much the same flavor reveals that if $\gamma \in G$, then

$$
f_{H}(\gamma \cdot(t, x))=f_{H}(t, x) .
$$

In other words $f_{H}$ is well defined on $\widehat{G} \times_{G} \widetilde{X}$. The collection $\left\{f_{H}\right\}$ is compatible with the inverse system of covering maps and therefore determines a continuous map

$$
\widehat{G} \times_{G} \tilde{X} \stackrel{f}{\longrightarrow} \mathbf{X}_{\infty} .
$$

The next claim is that $f$ is injective. We will use the subgroups $A_{n}$ discussed in Section 2.3. Suppose $(t, x)$ and $(s, y)$ represent elements of $\widehat{G} \times_{G} \widetilde{X}$ that are mapped by $f$ to the same point in $\mathbf{X}_{\infty}$. Since $f_{G}$ maps both elements to the same point in $X_{G}$ we may assume $x=y$. By hypothesis, for every $n$ there exist $\gamma_{n}$ and $\zeta_{n}$ in $G$ such that

- $t \gamma_{n}^{-1}$ and $s \zeta_{n}^{-1}$ both lie in $\widehat{A}_{n}$, and

- $\gamma_{n} x$ and $\zeta_{n} x$ are projected onto the same point in $A_{n} \backslash \widetilde{X}$.

The second condition implies $\zeta_{n} \gamma_{n}^{-1} \in A_{n}$. For every $n$,

$$
\left(s \zeta_{n}^{-1}\right)^{-1}\left(t \gamma_{n}^{-1}\right)=\zeta_{n} s^{-1} t \gamma_{n}^{-1}=\left(\zeta_{n} s^{-1} t \zeta_{n}^{-1}\right) \zeta_{n} \gamma_{n}^{-1} .
$$

Since $\widehat{A}_{n}$ is normal in $\widehat{G}$ it follows that $s^{-1} t \in \widehat{A}_{n}$; therefore, $s=t$ and $f$ is injective.

We merely sketch the argument for surjectivity. Let $z$ be an arbitrary point in $\mathbf{X}_{\infty}$ and let $n$ be a positive integer. Project $z$ onto the surface $A_{n} \backslash \widetilde{X}$ and take the full inverse image of this point in $\widetilde{X}$, thereby obtaining an $A_{n}$-invariant collection 
of points in $\widetilde{X}$. From this collection choose a point $z_{n}$. Define $t_{n}$ to be the unique element of $\pi_{1}(X)$ that maps $z_{1}$ to $z_{n}$. The collection $\left\{t_{n}\right\}$ determines an element $t$ in $\widehat{\pi_{1}}(X)$ and $f\left(t, z_{1}\right)=z$.

Using the previous theorem we can easily discern some features of $\mathbf{X}_{\infty}$; for instance, $\mathbf{X}_{\infty}$ is a principal $\widehat{\pi_{1}}(X)$-bundle, and the leaf space of $\mathbf{X}_{\infty}$ is homeomorphic to $\widehat{\pi_{1}}(X) / \pi_{1}(X)$.

The identification of $\widetilde{X}$ and $\{1\} \times \widetilde{X}$ induces a canonical marked baseleaf that is consistent with the canonical marked baseleaf in $\mathbf{X}_{\infty}$. Put another way there is a commutative diagram

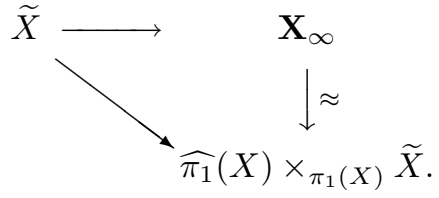

We pause to examine the subspace topology of $\widetilde{X}$ in $\mathbf{X}_{\infty}$. (Recall that we are using path classes in $(X, *)$ to construct the canonical model of $\widetilde{X}$.) Let $H$ be a subgroup of finite index in $\pi_{1}(X, *)$, let $\alpha$ be a path class based at $*$, and let $W$ be a contractible neighborhood of the terminus of $\alpha$. Define $N(\alpha, W, H)$ to be the collection of path classes that contain paths of the form $\gamma \cdot \alpha \cdot \beta$, where $\gamma$ represents an element of $H$ and $\beta$ is a path whose origin coincides with the terminus of $\alpha$ and whose image lies entirely in $W$. The sets $N(\alpha, W, H)$ comprise a base for the subspace topology of $\tilde{X}$. (Notice that this construction makes sense for arbitrary subgroups; indeed, when $H$ is trivial the standard topology on $\widetilde{X}$ is recovered.)

3.2. Models of solenoids. A covering solenoid is a complicated object; however, it is the quotient of a nice product space by the action of a reasonable group. A useful way to create a homeomorphism between covering solenoids is to construct an equivariant homeomorphism between representative product spaces.

Proposition 3.6. Let $(X, *)$ be a closed, pointed surface and let $H$ be a finite index subgroup of $G=\pi_{1}(X, *)$. There is a a commutative diagram of canonical maps

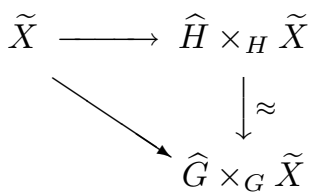

where the vertical map is a homeomorphism and the other maps are canonical baseleaf markings.

Proof. As in Theorem 3.5 it suffices to construct a continuous bijection. Define a map

$$
\widehat{H} \times \widetilde{X} \longrightarrow \widehat{G} \times_{G} \widetilde{X}
$$

by including $\widehat{H} \times \widetilde{X}$ in $\widehat{G} \times \widetilde{X}$ in the obvious fashion and taking the quotient by $G$. Suppose $(t, x)$ and $(s, y)$ have the same image; that is, there exists $\gamma$ in $G$ such that

$$
(t, x)=\gamma \cdot(s, y)=\left(s \gamma^{-1}, \gamma y\right)
$$


Writing $\gamma=t^{-1} s$ we find that $\gamma$ is in the intersection of $\widehat{H}$ and $G$, which is just $H$. It follows that there is a well defined, injective map

$$
\widehat{H} \times_{H} \widetilde{X} \longrightarrow \widehat{G} \times_{G} \widetilde{X} .
$$

A consequence of Lemma 2.16 is that this map is also surjective.

The canonical baseleaf markings are induced by the formula $x \mapsto(1, x)$ and the homeomorphism commutes with these baseleaf markings.

Theorem 3.7. Let $(X, *)$ be a pointed, compact surface; let $H$ and $K$ be subgroups of finite index in $G=\pi_{1}(X)$. If $\psi$ is a homeomorphism of $\widehat{H} \times \widetilde{X}$ onto $\widehat{K} \times \widetilde{X}$ that is equivariant with respect to $H$ and $K$, then $\psi$ canonically induces a selfhomeomorphism of $\mathbf{X}_{\infty}$.

Proof. We have the following commutative diagram of homeomorphisms:

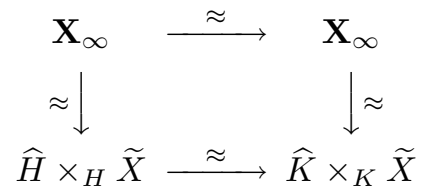

The vertical arrows exist thanks to Proposition 3.6. The equivariant map $\psi$ induces the homeomorphism of $\widehat{H} \times_{H} \widetilde{X}$ onto $\widehat{K} \times_{K} \widetilde{X}$. By pulling this homeomorphism back via the vertical maps we obtain the desired self-homeomorphism of $\mathbf{X}_{\infty}$.

The following proposition is an application of Theorem [3.7] Its proof includes the prototypical calculation used to verify equivariance.

Proposition 3.8. Let $(X, *)$ be a pointed, compact surface, and let $H$ and $K$ be subgroups of finite index in $G=\pi_{1}(X)$. If $f$ is a basepoint respecting homeomorphism of the surface $X_{H}$ onto the surface $X_{K}$, then $f$ induces a basepoint preserving self-homeomorphism of $\mathbf{X}_{\infty}$.

Proof. By Theorem 3.7 it is enough to show that $f$ induces an equivariant homeomorphism of $\widehat{H} \times \widetilde{X}$ onto $\widehat{K} \times \widetilde{X}$. Let $f_{*}$ be the isomorphism of $\widehat{H}$ onto $\widehat{K}$ induced by $f$ and let $\tilde{f}$ be the unique basepoint preserving self-homeomorphism of $\widetilde{X}$ that covers $f$. Define the map $\psi$ by the rule

$$
(t, x) \stackrel{\psi}{\longmapsto}\left(f_{*}(t), \tilde{f}(x)\right) .
$$

It is obvious that $\psi$ is a homeomorphism of $\widehat{H} \times \widetilde{X}$ onto $\widehat{K} \times \widetilde{X}$. To show that $\psi$ is equivariant we exploit the fact that $\widetilde{f}$ is an equivariant homeomorphism of $\widetilde{X}$; that is, if $h \in H$, then

$$
\tilde{f} h \tilde{f}^{-1}=f_{*}(h) .
$$

All that remains is a routine calculation:

$$
\begin{aligned}
\psi(h \cdot(t, x)) & =\psi\left(t h^{-1}, h x\right) \\
& =\left(f_{*}\left(t h^{-1}\right), \tilde{f}(h x)\right) \\
& =\left(f_{*}(t) f_{*}(h)^{-1}, f_{*}(h) \cdot \tilde{f}(x)\right) \\
& =f_{*}(h) \cdot \psi(t, x) .
\end{aligned}
$$


3.3. The $G$-tagged solenoid. In this section we introduce the $G$-tagged solenoid, a covering solenoid that inherits complex and geometric structures from a Fuchsian group $G$. These structures will be exploited in Section 4 to study the mapping class group of the $G$-tagged solenoid.

Definition 3.9. Let $G$ be a cocompact Fuchsian group. The $G$-tagged hyperbolic solenoid is the quotient

$$
\mathbf{H}_{\infty}^{G} \stackrel{\text { def }}{=} \widehat{G} \times_{G} \mathbf{D} .
$$

The $G$-tagged solenoid has no canonical basepoint; however, the identification of $\mathbf{D}$ and $\{1\} \times \mathbf{D}$ induces a canonical marked baseleaf. Clearly this marking amounts to recognizing the identity element in $\widehat{G}$. To forget this baseleaf is equivalent to recognizing only the structure of $\widehat{G}$ as a homogeneous space for the action of $G$.

An understanding of $G$-tagged solenoids depends on the Teichmüller theory of the universal hyperbolic solenoid. This theory was introduced in [21] and 7], and developed extensively in [18, [3], 4], 5], and [2]. A brief resumé of this theory is given below. To fix notation and help set the stage for this discussion we will quickly review the Teichmüller theory of surfaces.

Let $S$ be a closed Riemann surface. A Teichmüller marked surface is a triple $[S, f, Y]$, where $Y$ is another Riemann surface and $f$ is a homeomorphism of $S$ onto $Y$. Two triples $[S, f, Y]$ and $[S, g, Z]$ are Teichmüller equivalent if there exists a biholomorphism $\Phi$ of $Y$ onto $Z$ such that $g^{-1} \circ \Phi \circ f$ is isotopic to the identity map on $S$. The Teichmüller space of $S$, denoted $T(S)$, is the set of equivalence classes of marked surfaces. This set is topologized via the Teichmüller metric $\tau$ defined by setting

$$
\tau([S, f, Y],[S, g, Z])=\inf \left\{\frac{1}{2} \log K(h) \mid h \text { is isotopic to } g \circ f^{-1}\right\},
$$

where $K(h)$ denotes the maximal dilatation of $h$.

By passing to the universal covering space we arrive at a topologically equivalent construction. Choose a Fuchsian group $G$ that uniformizes $S$. Let $Q C(\mathbf{D})$ denote the collection of quasiconformal self-homeomorphisms of D. An element $f$ of $Q C(\mathbf{D})$ is said to be $G$-compatible if $f G f^{-1}$ is a Fuchsian group. Let $Q C(\mathbf{D}, G)$ denote the collection of $G$-compatible mappings. As a set the Teichmüller space $T(G)$ is defined to be

$$
T(G) \stackrel{\text { def }}{=} \operatorname{Möb}(\mathbf{D}) \backslash Q C(\mathbf{D}, G) / \text { isotopy rel } \partial \mathbf{D} .
$$

This set is topologized by defining a Teichmüller metric analogous to the one introduced above. If $G$ is trivial we obtain the universal Teichmüller space

$$
T(\mathbf{D}) \stackrel{\text { def }}{=} \operatorname{Möb}(\mathbf{D}) \backslash Q C(\mathbf{D}) / \text { isotopy rel } \partial \mathbf{D} \text {. }
$$

The space $T(\mathbf{D})$ deserves its name in view of the fact that it contains the Teichmüller space of every hyperbolic Riemann surface.

A complex structure on an arbitrary two-solenoid is a maximal atlas of continuous charts onto sets of the form $U \times D$, where $U$ is an open subset of the complex plane, $D$ is a totally disconnected space, and the transition functions are required to be holomorphic on leafs [21]. A Teichmüller marked solenoid is a triple $\left[\mathbf{H}_{\infty}^{G}, f, Y\right]$, where $f$ is a homeomorphism of $\mathbf{H}_{\infty}^{G}$ onto a solenoid $Y$ that is equipped with a complex structure. The marked solenoids $\left[\mathbf{H}_{\infty}^{G}, f, Y\right]$ and $\left[\mathbf{H}_{\infty}^{G}, g, Z\right]$ are Teichmüller equivalent if there is a homeomorphism $\Phi$ from $Y$ onto $Z$ such that $\Phi$ is locally biholomorphic on leaves and $g^{-1} \circ \Phi \circ f$ is isotopic to the identity homeomorphism 
of $\mathbf{H}_{\infty}^{G}$. By definition the Teichmüller space $T\left(\mathbf{H}_{\infty}^{G}\right)$ is the collection of equivalence classes of marked solenoids.

A complex structure on $\mathbf{H}_{\infty}^{G}$ induces a complex structure on the baseleaf $\mathbf{D}$. In this way one obtains an inclusion of $T\left(\mathbf{H}_{\infty}^{G}\right)$ in $T(\mathbf{D})$. A complex structure on the surface $G \backslash \mathbf{D}$ can be lifted to a "transversely locally constant" structure on $\mathbf{H}_{\infty}^{G}$. For every subgroup $K$ of finite index in $G$ there are inclusions

$$
T(K) \subset T\left(\mathbf{H}_{\infty}^{G}\right) \subset T(\mathbf{D}) .
$$

The transversely locally constant structures are dense in $T\left(\mathbf{H}_{\infty}^{G}\right)$; in other words,

$$
T\left(\mathbf{H}_{\infty}^{G}\right)=\overline{\bigcup_{H<G} T(H)} \subset T(\mathbf{D})
$$

where closure is taken with respect to the Teichmüller metric in $T(\mathbf{D})$ [7].

The following proposition describes the explicit passage from a Teichmüller marked surface to a Teichmüller marked solenoid.

Proposition 3.10. If $f$ is a quasiconformal self-homeomorphism of $\mathbf{D}$ that is $G$ compatible, then $f$ induces a homeomorphism

$$
\mathbf{H}_{\infty}^{G} \stackrel{\approx}{\longrightarrow} \mathbf{H}_{\infty}^{f G f^{-1}} .
$$

Proof. Let $f_{*}$ be the isomorphism of $\widehat{G}$ onto $\widehat{f G f^{-1}}$ that is induced by $f$. The formula $(t, x) \mapsto\left(f_{*}(t), f(x)\right)$ defines an equivariant homeomorphism of $\widehat{G} \times \mathbf{D}$ onto $\widehat{f G f^{-1}} \times \mathbf{D}$ which in turn defines a homeomorphism of $\mathbf{H}_{\infty}^{G}$ onto $\mathbf{H}_{\infty}^{f G f^{-1}}$.

We will now construct a distance function on the $G$-tagged solenoid that serves an important auxiliary role in the next section. Let $G$ be a cocompact Fuchsian group, let $\rho$ be the profinite distance function on $G$ discussed in Section 2.3 and let $d$ be the standard distance function on $\mathbf{D}$ that arises from the Poincaré metric. On the product $\widehat{G} \times \mathbf{D}$ define the distance function

$$
\sigma((t, x),(s, y)) \stackrel{\text { def }}{=} \max \{\rho(t, s), d(x, y)\} .
$$

It is clear that the action of $G$ preserves $\sigma$ and that $\sigma$ descends to a metric on the quotient space $\mathbf{H}_{\infty}^{G}$. Explicitly, let $z_{1}, z_{2}$ be points in $\mathbf{H}_{\infty}^{G}$, and let $\tilde{z_{1}}, \tilde{z_{2}}$ be elements of $\widehat{G} \times \mathbf{D}$ that represent these points. Define

$$
\sigma_{G}\left(z_{1}, z_{2}\right) \stackrel{\text { def }}{=} \inf _{\gamma, \zeta \in G}\left\{\sigma\left(\gamma \tilde{z_{1}}, \zeta \tilde{z_{2}}\right)\right\} .
$$

Evidently $\sigma_{G}$ does not depend on the choice of $\tilde{z_{1}}$ and $\tilde{z_{2}}$. It is straightforward to verify that $\sigma_{G}$ is a metric on $\mathbf{H}_{\infty}^{G}$.

A ball of small radius in $\mathbf{H}_{\infty}^{G}$ looks like the product of balls in $\widehat{G}$ and $\mathbf{D}$. To be precise, suppose that $\epsilon$ is smaller than the injectivity radius of the surface $G \backslash \mathbf{D}$. Let $P$ denote the canonical projection of $\mathbf{H}_{\infty}^{G}$ onto $G \backslash \mathbf{D}$. Choose $z$ in $\mathbf{H}_{\infty}^{G}$ and set $z_{0}=P(z)$. The $\epsilon$-ball centered at $z$, denoted $B_{\epsilon}(z)$, projects onto the $\epsilon$-disk $D_{\epsilon}\left(z_{0}\right)$ in $G \backslash \mathbf{D}_{G}$ centered at $z_{0}$. There is a finite index subgroup $\widehat{H}$ whose right cosets in $\widehat{G}$ are the $\epsilon$-balls in $\widehat{G}$; hence, $B_{\epsilon}(z)$ is isometric to $\widehat{H} \times D_{\epsilon}\left(z_{0}\right)$. Notice that the "central fiber" in $B_{\epsilon}(z)$ coincides with the "centers" of $B_{\epsilon}(z)$; that is, if $P\left(z^{\prime}\right)=z_{0}$, then $B_{\epsilon}\left(z^{\prime}\right)=B_{\epsilon}(z)$. The intersection of an $\epsilon$-ball and a leaf is a disjoint collection of $\epsilon$-disks that are invariant under the action of some subgroup of finite index in $G$.

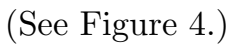




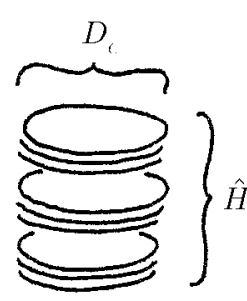

$B$

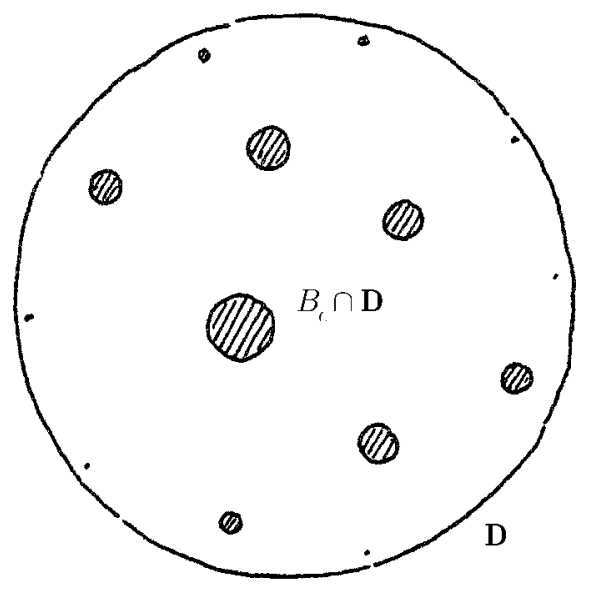

Figure 4. An $\epsilon$-ball in $\mathbf{H}_{\infty}^{G}$.

An isometry of $\mathbf{H}_{\infty}^{G}$ is a self-homeomorphism that is isometric on leafs. (Notice the absence of any requirement to preserve metric structure in the transverse direction.) Let $\operatorname{Isom}\left(\mathbf{H}_{\infty}^{G}\right)$ denote the isometry group of $\mathbf{H}_{\infty}^{G}$, and let $\operatorname{Isom}_{B L P}^{+}\left(\mathbf{H}_{\infty}^{G}\right)$ denote the subgroup of orientation preserving, baseleaf preserving isometries.

Example 3.11. There is a canonical, injective homomorphism

$$
\operatorname{Comm}_{\operatorname{Möb}(\mathbf{D})} G \longrightarrow \operatorname{Isom}_{B L P}^{+}\left(\mathbf{H}_{\infty}^{G}\right) \text {. }
$$

Suppose $\gamma$ is a Möbius element, $H$ and $K$ are finite index subgroups of $G$, and $\gamma H \gamma^{-1}=K$. Let $\gamma_{*}$ be the induced isomorphism of $\widehat{H}$ onto $\widehat{K}$. The equivariant homeomorphism

$$
(t, x) \stackrel{\psi_{\gamma}}{\longmapsto}\left(\gamma_{*}(t), \gamma x\right)
$$

of $\widehat{H} \times \mathbf{D}$ onto $\widehat{K} \times \mathbf{D}$ defines a self-homeomorphism of $\mathbf{H}_{\infty}^{G}$. It is easy to check that the assignment $\gamma \mapsto \psi_{\gamma}$ induces an injective homomorphism of $\operatorname{Comm}_{\mathrm{Möb}(\mathbf{D})} G$ into $\operatorname{Isom}_{B L P}^{+}\left(\mathbf{H}_{\infty}^{G}\right)$.

Example 3.12. There is a canonical, injective homomorphism

$$
\widehat{G} \longrightarrow \operatorname{Isom}\left(\mathbf{H}_{\infty}^{G}\right) \text {. }
$$

For every $s$ in $\widehat{G}$, define the self-homeomorphism $\phi_{s}$ of $\widehat{G} \times \mathbf{D}$ by the formula

$$
(t, x) \stackrel{\phi_{s}}{\longmapsto}(s t, x) .
$$

This map is trivially equivariant with respect to the action of $G$ and the assignment $s \mapsto \phi_{s}$ induces an injective homomorphism of $\widehat{G}$ into $\operatorname{Isom}\left(\mathbf{H}_{\infty}^{G}\right)$.

Examples 3.11 and 3.12 provide two injective maps of $G$ into $\operatorname{Isom}\left(\mathbf{H}_{\infty}^{G}\right)$. These two maps are in fact the same, for if $\gamma \in G$, then

$$
\psi_{\gamma}(t, x)=\left(\gamma t \gamma^{-1}, \gamma x\right)=\gamma \cdot(\gamma t, x)=\gamma \cdot \phi_{\gamma}(t, x) .
$$

It follows that $\psi_{\gamma}$ and $\phi_{\gamma}$ induce the same isometry of $\mathbf{H}_{\infty}^{G}$.

A hyperbolic surface has very few isometries; in fact, the isometry group of such a surface is finite. A $G$-tagged solenoid, however, can have many isometries. 
Example 3.12 reveals that the full isometry group of $\mathbf{H}_{\infty}^{G}$ always contains a profinite group. Even the baseleaf preserving isometry group can be large. If $G$ is arithmetic, then $\operatorname{Comm}_{\operatorname{Möb}(\mathbf{D})} G$ is dense in $\operatorname{Möb}(\mathbf{D})[22]$. It follows that $\operatorname{Isom}_{B L P}^{+}\left(\mathbf{H}_{\infty}^{G}\right)$ is dense in $\operatorname{Möb}(\mathbf{D})$.

Remark. There is a natural measure on $\mathbf{H}_{\infty}^{G}$ that is induced by the product of Haar measure on $\widehat{G}$ and the standard measure on $\mathbf{D}$. Biswas and Nag [6] proved that the action of $\operatorname{Isom}_{B L P}\left(\mathbf{H}_{\infty}^{G}\right)$ is ergodic if and only if $G$ is arithmetic.

In Section 4.3 it will be proved that every isometry of $\mathbf{H}_{\infty}^{G}$ is a composition of isometries found in Examples 3.11 and 3.12 .

\section{MAPPING CLASS GROUPS}

4.1. Self-homeomorphisms of $\mathbf{X}_{\infty}$. In this section we examine the group of selfhomeomorphisms of $\mathbf{X}_{\infty}$. We begin with the following definitions:

$$
\begin{aligned}
& \operatorname{Homeo}\left(\mathbf{X}_{\infty}\right)=\text { the group of self-homeomorphisms of } \mathbf{X}_{\infty} \text {, } \\
& \text { Homeo }_{B L P}\left(\mathbf{X}_{\infty}\right)=\text { the subgroup of self-homeomorphisms } \\
& \text { that preserve the canonical baseleaf, } \\
& \text { Homeo }_{0}\left(\mathbf{X}_{\infty}\right) \quad=\text { the subgroup of isotopically trivial } \\
& \text { self-homeomorphisms. }
\end{aligned}
$$

It is easily verified that $\mathrm{Homeo}_{0}\left(\mathbf{X}_{\infty}\right)$ is normal in both $\mathrm{Homeo}_{B L P}\left(\mathbf{X}_{\infty}\right)$ and $\operatorname{Homeo}\left(\mathbf{X}_{\infty}\right)$.

Example 4.1. The natural action of $\widehat{\pi}_{1}(X)$ on itself generates self-homeomorphisms of $\mathbf{X}_{\infty}$. Given $s \in \widehat{\pi_{1}}(X)$ define the map $T_{s}$ by the rule

$$
(t, x) \stackrel{T_{s}}{\longmapsto}(s t, x) .
$$

The map $s \mapsto T_{s}$ induces an injective homomorphism

$$
\widehat{\pi_{1}}(X) \longrightarrow \operatorname{Homeo}\left(\mathbf{X}_{\infty}\right) \text {. }
$$

(This is the topological version of Example 3.12.)

Example 4.2. The covering action of $\pi_{1}(X)$ on $\widetilde{X}$ generates baseleaf preserving homeomorphisms in the following manner. For every $\gamma$ in $\pi_{1}(X)$ define the equivariant self-homeomorphism $C_{\gamma}$ of $\widehat{\pi}_{1}(X) \times \widetilde{X}$ by the formula

$$
(t, x) \stackrel{C_{\gamma}}{\longmapsto}\left(\gamma t \gamma^{-1}, \gamma x\right) .
$$

The assignment $\gamma \mapsto C_{\gamma}$ induces an injective homomorphism

$$
\pi_{1}(X) \longrightarrow \text { Homeo }_{B L P}\left(\mathbf{X}_{\infty}\right) \text {. }
$$

Remark. As was the case with Examples 3.11 and 3.12 there is some overlap in these examples. If $\gamma \in \pi_{1}(X)$, then

$$
C_{\gamma}(t, x)=\left(\gamma t \gamma^{-1}, \gamma x\right)=\gamma \cdot(\gamma t, x)=\gamma \cdot T_{\gamma}(t, x) .
$$

From now on, for every $s$ in $\widehat{\pi_{1}}(X)$ let $T_{s}$ denote the homeomorphism induced by $s$, and for every $\gamma$ in $\pi_{1}(X)$ let $C_{\gamma}$ denote the baseleaf preserving homeomorphism induced by the covering action of $\gamma$. It follows that if $\gamma \in \pi_{1}(X)$, then $C_{\gamma}=T_{\gamma}$. This redundancy of notation will turn out to be useful. 
Example 4.3. By taking $H=K$ in Proposition 3.8 it follows that the set of basepoint preserving self-homeomorphisms of the surface $X_{H}$ may be naturally regarded as a subgroup of $\mathrm{Homeo}_{B L P}\left(\mathbf{X}_{\infty}\right)$.

Each leaf in $\mathbf{X}_{\infty}$ is naturally identified with a coset in $\widehat{\pi_{1}}(X) / \pi_{1}(X)$. The action here is defined in the following way: for every $\gamma$ in $\pi_{1}(X)$ and every $t$ in $\widehat{\pi_{1}}(X)$, set

$$
\gamma \cdot t=t \gamma^{-1}
$$

Let $[t]$ denote the coset that contains $t$.

It is trivially the case that $\widehat{\pi_{1}}(X)$ acts transitively on leafs; however, it does not act simply. The homeomorphism induced by $s$ fixes the leaf $[t]$ if and only if there exists $\gamma$ in $\pi_{1}(X)$ such that $s t=t \gamma^{-1}$. It follows that $t \pi_{1}(X) t^{-1}$ is the isotropy subgroup -in $\widehat{\pi_{1}}(X)$ - of $[t]$. In particular

$$
\widehat{\pi_{1}}(X) \cap \operatorname{Homeo}_{B L P}\left(\mathbf{X}_{\infty}\right)=\pi_{1}(X) .
$$

Define an action of $\pi_{1}(X)$ on the product space

$$
\widehat{\pi_{1}}(X) \times \operatorname{Homeo}_{B L P}\left(\mathbf{X}_{\infty}\right)
$$

by the formula

$$
\gamma \cdot\left(T_{s}, \phi\right) \stackrel{\text { def }}{=}\left(T_{s} \circ T_{\gamma}^{-1}, C_{\gamma} \circ \phi\right) .
$$

The following theorem asserts that the resulting quotient space is homeomorphic to the topological group $\operatorname{Homeo}\left(\mathbf{X}_{\infty}\right)$.

Theorem 4.4. There is a canonical homeomorphism

$$
\widehat{\pi_{1}}(X) \times_{\pi_{1}(X)} \operatorname{Homeo}_{B L P}\left(\mathbf{X}_{\infty}\right) \stackrel{\approx}{\longrightarrow} \operatorname{Homeo}\left(\mathbf{X}_{\infty}\right) .
$$

Proof. Note $\widehat{\pi}_{1}(X) \times \operatorname{Homeo}_{B L P}\left(\mathbf{X}_{\infty}\right)$ is a subspace of $\operatorname{Homeo}\left(\mathbf{X}_{\infty}\right) \times \operatorname{Homeo}\left(\mathbf{X}_{\infty}\right)$. Composition defines a continuous map into $\operatorname{Homeo}\left(\mathbf{X}_{\infty}\right)$. The claim is that this map induces the desired homeomorphism.

To prove surjectivity let $f$ be an arbitrary self-homeomorphism of $\mathbf{X}_{\infty}$. Since $\widehat{\pi_{1}}(X)$ acts transitively on leafs there exists some $s$ in $\widehat{\pi_{1}}(X)$ such that

$$
T_{s} \circ f \in \text { Homeo }_{B L P}\left(\mathbf{X}_{\infty}\right) \text {. }
$$

Setting $\psi=T_{s} \circ f$ we can express $f$ as a product $T_{s^{-1}} \circ \psi$, where $\psi$ is a baseleaf preserving homeomorphism. This proves surjectivity.

Suppose there is another such expression $f=T_{u^{-1}} \circ \phi$. It follows that

$$
T_{s u^{-1}}=T_{s} \circ T_{u^{-1}}=\psi \circ \phi^{-1} .
$$

The right-hand side is a baseleaf preserving homeomorphism; therefore, $s u^{-1} \in$ $\pi_{1}(X)$. Setting $\gamma=s u^{-1}$ we have

$$
f=T_{u^{-1}} \circ \phi=T_{s^{-1} \gamma} \circ \phi=T_{s^{-1}} \circ\left(C_{\gamma} \circ \phi\right)
$$

and conclude

$$
u^{-1}=s^{-1} \gamma \quad \text { and } \quad \psi=C_{\gamma} \circ \phi
$$

It follows that the induced map of the quotient space $\widehat{\pi_{1}}(X) \times_{\pi_{1}(X)} \operatorname{Homeo}_{B L P}\left(\mathbf{X}_{\infty}\right)$ onto the full homeomorphism group is bijective. By tracing through the maps and using the definition of the quotient topology it is easily proved that this map is in fact a homeomorphism. 
4.2. The baseleaf preserving mapping class group of $\mathbf{H}_{\infty}^{G}$. In this section we will restrict our attention to $G$-tagged solenoids. By definition the baseleaf preserving mapping class group is the group of isotopy classes of baseleaf preserving self-homeomorphisms of $\mathbf{H}_{\infty}^{G}$. We will prove (Theorem 4.7) that this group is naturally isomorphic to the virtual automorphism group of $G$, a result which first appeared in [20]. This theorem is not surprising in view of [7], where it was proved that the virtual automorphism group acts as a modular group on $T\left(\mathbf{H}_{\infty}^{G}\right)$.

Let $\Gamma_{B L P}\left(\mathbf{H}_{\infty}^{G}\right)$ denote the baseleaf preserving mapping class group of $\mathbf{H}_{\infty}^{G}$; that is,

$$
\Gamma_{B L P}\left(\mathbf{H}_{\infty}^{G}\right) \stackrel{\text { def }}{=} \operatorname{Homeo}_{B L P}\left(\mathbf{H}_{\infty}^{G}\right) / \operatorname{Homeo}_{0}\left(\mathbf{H}_{\infty}^{G}\right) .
$$

In order to establish the isomorphism

$$
\Gamma_{B L P}\left(\mathbf{H}_{\infty}^{G}\right) \stackrel{\cong}{\longrightarrow} \operatorname{Vaut}(G)
$$

we will use the natural action of $\operatorname{Vaut}(G)$ on $\partial \mathbf{D}$, the boundary of the marked baseleaf.

Proposition 4.5. There is a natural injective homomorphism

$$
\operatorname{Vaut}(G) \longrightarrow \operatorname{Homeo}(\partial \mathbf{D})
$$

whose image is $\operatorname{Comm}_{\operatorname{Homeo}(\partial \mathbf{D})} G$.

Proof. Nielsen proved that if $H$ and $K$ are Fuchsian groups and if $\phi$ is an isomorphism of $H$ onto $K$, then there exists a self-homeomorphism defined on the closure of $\mathbf{D}$ that conjugates the action of $H$ to the action of $K$ [19]. Although this homeomorphism is not unique its restriction to $\partial \mathbf{D} i s$ completely determined by $\phi$ as we shall see in a moment.

Let $\phi$ be an isomorphism of $H$ onto $K$, both of which are subgroups of index $n$ in $G$. The goal is to construct a self-homeomorphism $\phi_{*}$ of $\partial \mathbf{D}$. If $\gamma \in G$, then $\gamma^{n !} \in H$. Let $\alpha(\gamma)$ (resp. $\omega(\gamma)$ ) denote the repelling (resp. attracting) fixed point of $\gamma$. Define $\phi_{*}$ by the rules

$$
\alpha(\gamma) \stackrel{\phi_{*}}{\longmapsto} \alpha\left(\phi\left(\gamma^{n !}\right)\right) \quad \text { and } \quad \omega(\gamma) \stackrel{\phi_{*}}{\longmapsto} \omega\left(\phi\left(\gamma^{n !}\right)\right) .
$$

Since the fixed points of $G$ are dense in $\partial \mathbf{D}, \phi_{*}$ is determined by its action on these points.

It is easily verified that equivalent partial automorphisms of $G$ induce the same homeomorphism of $\partial \mathbf{D}$; therefore, the assignment $\phi \mapsto \phi_{*}$ defines a homomorphism of $\operatorname{Vaut}(G)$ into Homeo $(\partial \mathbf{D})$.

The injectivity of this map is proved by considering transversely intersecting axes of $G$. Suppose $\phi$ is a partial automorphism of $G$ that induces the identity map on $\partial \mathbf{D}$. This implies $\phi$ preserves power classes of $G$ - precisely, for every $\gamma$ in $G$ there exist $m$ and $n$ such that $\phi\left(\gamma^{m}\right)=\gamma^{n}$. We wish to show that $m=n$ for every $\gamma$. To obtain a contradiction suppose that $\phi\left(\gamma^{m}\right)=\gamma^{n}$ and $m \neq n$. Choose an element $\zeta$ whose axis intersects the axis of $\gamma$ transversely. There exists some $k$ for which

$$
\phi\left(\gamma^{m} \zeta^{k} \gamma^{-m}\right)=\gamma^{n} \phi\left(\zeta^{k}\right) \gamma^{-n} .
$$

Since the fixed points of $\gamma^{m} \zeta^{k} \gamma^{-m}$ and $\gamma^{n} \phi\left(\zeta^{k}\right) \gamma^{-n}$ are distinct, it follows that $\phi_{*}$ acts nontrivially on $\partial \mathbf{D}$, a contradiction. 
Theorem 4.6. There is a natural homomorphism

$$
\operatorname{Homeo}_{B L P}\left(\mathbf{H}_{\infty}^{G}\right) \longrightarrow \operatorname{Homeo}(\partial \mathbf{D})
$$

whose image is $\operatorname{Comm}_{\operatorname{Homeo}(\partial \mathbf{D})} G$. Consequently there is a natural homomorphism

$$
\operatorname{Homeo}_{B L P}\left(\mathbf{H}_{\infty}^{G}\right) \longrightarrow \operatorname{Vaut}(G) .
$$

Proof. Let $f$ be a baseleaf preserving self-homeomorphism of $\mathbf{H}_{\infty}^{G}$. The goal is to associate to $f$ a partial automorphism of $G$ (denoted $\phi_{f}$ ) and prove that $f$ induces a self-homeomorphism of $\partial \mathbf{D}$ that agrees with the action of $\phi_{f}$ there.

The first step is to find a subgroup of finite index in $G$ on which $\phi_{f}$ will be defined. To this end let $\epsilon$ be a positive number that is smaller than one-fourth the injectivity radius of the surface $G \backslash \mathbf{D}$. Since $\mathbf{H}_{\infty}^{G}$ is compact, $f$ is uniformly continuous. Choose a positive number $\delta$ so that $\delta<\epsilon$ and

$$
\sigma_{G}(x, y)<\delta \text { implies } \sigma_{G}(f(x), f(y))<\epsilon .
$$

As described in Section 2.3 let $H$ and $K$ be the subgroups of $G$ that correspond to the numbers $\delta$ and $\epsilon$. The subgroup $H$ will turn out to be the domain of $\phi_{f}$.

Cover the surface $G \backslash \mathbf{D}$ by a finite collection of $\delta$-disks and lift these to a collection $\left\{B_{1}, \ldots, B_{N}\right\}$ of $\delta$-balls that cover $\mathbf{H}_{\infty}^{G}$. Consider the intersection of the baseleaf $\mathbf{D}$ and some ball $B_{j}$. Each path connected component of this set is a $\delta$-disk in $\mathbf{D}$; the collection of these disk components is invariant under the action of $H$. Label each such disk $\Delta_{j}^{i}$. The subscript $j$ ranges over the finite set $\{1, \ldots, N\}$ and indicates that $\Delta_{j}^{i}$ is a component of $B_{j}$. For fixed $j$ the superscript $i$ ranges over an index set with the cardinality of $H$; indeed, the group $H$ acts simply transitively on the disk components of $B_{j} \cap \mathbf{D}$. We will exploit this fact in the following way: for every $h$ in $H$ let $\Delta_{j}^{h \cdot i}$ denote the disk $h\left(\Delta_{j}^{i}\right)$.

From among the centers of all disks $\left\{\Delta_{j}^{i}\right\}$ choose a collection $\left\{c_{1}, \ldots, c_{N}\right\}$ so that $c_{j}$ is a center of the ball $B_{j}$. Now set $c_{j}^{\prime}=f\left(c_{j}\right)$ and define $B_{j}^{\prime}$ to be the $\epsilon$-ball in $\mathbf{H}_{\infty}^{G}$ centered at $c_{j}^{\prime}$. The uniform continuity of $f$ implies $f\left(B_{j}\right) \subset B_{j}^{\prime}$. The intersection of $B_{j}^{\prime}$ and $\mathbf{D}$ is a $K$-invariant collection of $\epsilon$-disks and each set $f\left(\Delta_{j}^{i}\right)$ is contained in one of these disks. Define $D_{j}^{i}$ to be the disk component of $B_{j}^{\prime} \cap \mathbf{D}$ that contains $f\left(\Delta_{j}^{i}\right)$; see Figure 5 Following the convention set forth above, let $D_{j}^{h \cdot i}$ denote the disk component of $B_{j}^{\prime}$ that contains $f\left(\Delta_{j}^{h \cdot i}\right)$.

Claim A. If $\Delta_{j}^{i_{0}} \neq \Delta_{j}^{i_{1}}$, then $D_{j}^{i_{0}} \neq D_{j}^{i_{1}}$.

Proof. To obtain a contradiction suppose $D=D_{j}^{i_{0}}=D_{j}^{i_{1}}$. There exists a simple path $\gamma:[0,1] \rightarrow \mathbf{D}$ such that

- $\gamma(0)$ is the center of $\Delta_{j}^{i_{0}}$,

- $\gamma(1)$ is the center of $\Delta_{j}^{i_{1}}$, and

- $f \circ \gamma$ lies entirely in $D$.

Let $h$ be the unique element of $H$ that maps $\Delta_{j}^{i_{0}}$ to $\Delta_{j}^{i_{1}}$. Define the path $\tilde{\gamma}: \mathbf{R} \rightarrow \mathbf{D}$ by extending $\gamma$ along the axis of $h$-precisely, for every $t$ in $[n, n+1)$ set

$$
\tilde{\gamma}(t)=h^{n}(\gamma(t))
$$

By uniform continuity of $f$ as well as the connectedness of $\tilde{\gamma}$, it follows that $f \circ \tilde{\gamma}$ lies in a single disk of radius $2 \epsilon$. But this is impossible, for $\mathbf{D}-\tilde{\gamma}$ is the disjoint union of simply connected regions while $f(\mathbf{D}-\tilde{\gamma})$ has a doubly connected component; see Figure 6 . 


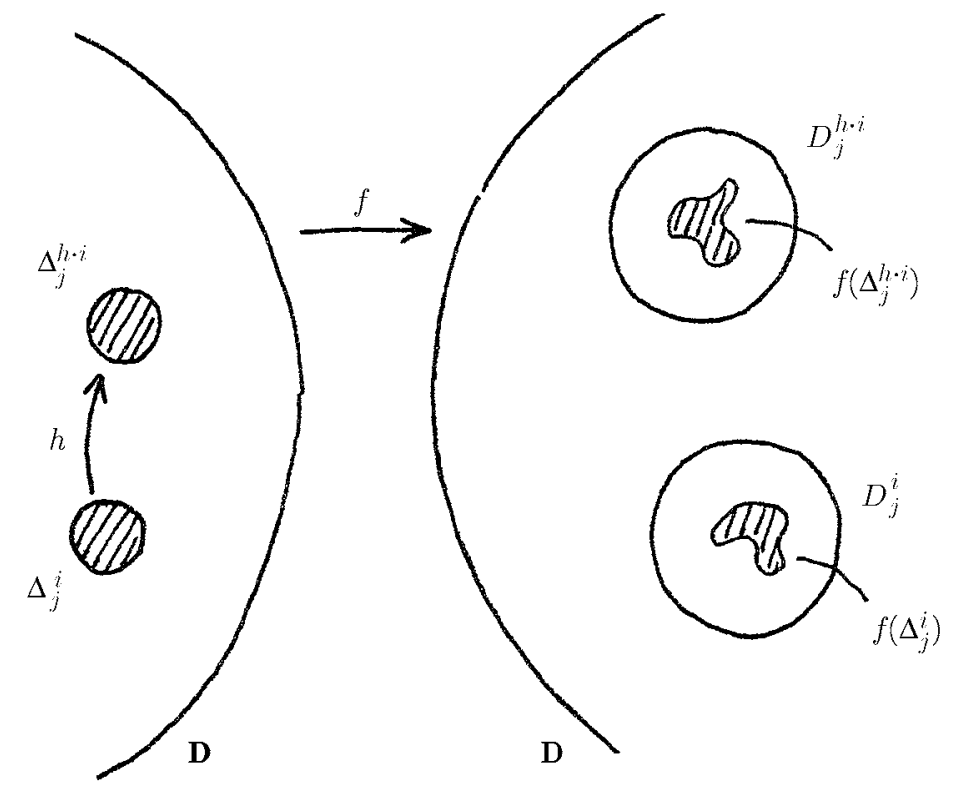

Figure 5. The disks $\Delta_{j}^{i}$ and $D_{j}^{i}$.

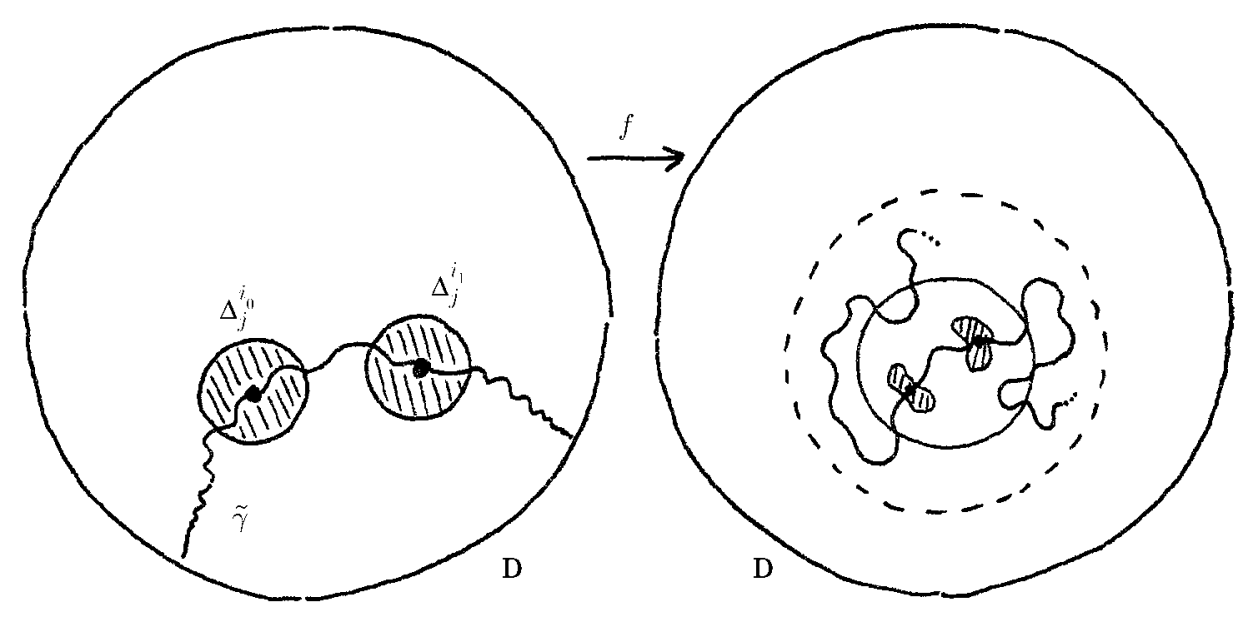

Figure 6. Proof of Claim A.

Since it was assumed that $\delta<\epsilon$, it is likely that $H$ is a proper subgroup of $K$ in which case the collection $\left\{D_{j}^{i}\right\}$ is a proper subset of the collection of disk components of $B_{j}^{\prime} \cap \mathbf{D}$. Notice, however, that $\bigcup_{i, j} D_{j}^{i}$ covers $\mathbf{D}$.

Let $h$ be an arbitrary element of $H$. We will prove that the homeomorphism $f h f^{-1}$ is uniformly approximated by an element of $K$. The first step is to show that this is locally true. To this end choose an arbitrary disk $\Delta_{j}^{i}$ and recall $f\left(\Delta_{j}^{i}\right) \subset D_{j}^{i}$. 
Let $k=k(i, j, h)$ be the unique element of $K$ such that

$$
k\left(D_{j}^{i}\right)=D_{j}^{h \cdot i} .
$$

For every $x$ in $f\left(\Delta_{j}^{i}\right)$,

$$
d\left(f h f^{-1}(x), k(x)\right)<2 \epsilon .
$$

In other words $k(i, j, h)$ approximates $f h f^{-1}$ uniformly on $f\left(\Delta_{j}^{i}\right)$. The next claim is that the function $k(i, j, h)$ is "locally constant".

Claim B. Given disks $\Delta_{j}^{i}$ and $\Delta_{j^{\prime}}^{i^{\prime}}$ and an element $h$ of $H$, let $k$ and $k^{\prime}$ be the uniquely determined elements of $K$ that satisfy

$$
k\left(D_{j}^{i}\right)=D_{j}^{h \cdot i} \quad \text { and } \quad k^{\prime}\left(D_{j^{\prime}}^{i^{\prime}}\right)=D_{j^{\prime}}^{h \cdot i^{\prime}} .
$$

If $\Delta_{j}^{i} \cap \Delta_{j^{\prime}}^{i^{\prime}}$ is not empty, then $k=k^{\prime}$.

Proof. It follows from the hypothesis that

$$
D_{j}^{i} \cap D_{j^{\prime}}^{i^{\prime}} \neq \emptyset \quad \text { and } \quad k\left(D_{j}^{i}\right) \cap k^{\prime}\left(D_{j^{\prime}}^{i^{\prime}}\right) \neq \emptyset .
$$

For some $x$ in $D_{j}^{i} \cup D_{j^{\prime}}^{i^{\prime}}$ the point $k^{-1} k^{\prime}(x)$ also lies in $D_{j}^{i} \cup D_{j^{\prime}}^{i^{\prime}}$; therefore, the distance between $x$ and $k^{-1} k^{\prime}(x)$ is bounded by $4 \epsilon$, which is smaller than the injectivity radius of $G \backslash \mathbf{D}$. This forces $k^{-1} k^{\prime}$ to be trivial.

Since $\mathbf{D}$ is connected, the function $k(i, j, h)$ is independent of $i$ and $j$. It follows that

$$
d\left(f h f^{-1}(x), k(x)\right)<2 \epsilon
$$

holds for every $x$ in $\mathbf{D}$. In other words $f h f^{-1}$ is uniformly approximated on $\mathbf{D}$ by the Möbius transformation $k$. An immediate consequence is that $f h f^{-1}$ extends to a self-homeomorphism of $\mathbf{D} \cup \partial \mathbf{D}$-indeed, the boundary homeomorphism induced by $f h f^{-1}$ is precisely the restriction of $k$ to $\partial \mathbf{D}$.

We will now prove that $f$ itself extends to a self-homeomorphism of $\mathbf{D} \cup \partial \mathbf{D}$. By the previous paragraph if $h$ is nontrivial, then $f h f^{-1}$ induces a nontrivial Möbius transformation on $\partial \mathbf{D}$. Choose an arbitrary point $x$ in $\mathbf{D}$. The sequence of points

$$
h^{n}(x), \quad n \geq 0,
$$

converges to the attracting fixed point of $h$. The uniform continuity of $f$ implies the sequence $\left\{f\left(h^{n}(x)\right)\right\}$ converges to the attracting fixed point of $k$. The fixed points of $H$ (as well as $K$ ) are dense in $\partial \mathbf{D}$; therefore, $f$ extends to a self-homeomorphism of $\mathbf{D} \cup \partial \mathbf{D}$.

Restricting our attention to the boundary, we notice the map $h \mapsto f h f^{-1}$ is an injective homomorphism of $H$ into $K$. Since $H$ has finite index in $G$, the image of $H$ under this map must also have finite index in $G$. It follows that

$$
f \in \operatorname{Comm}_{\text {Homeo( }(\partial \mathbf{D})} G .
$$

Setting $\phi_{f}$ equal to the partial automorphism of $G$ induced by $f$, we note that the assignment $f \mapsto\left[\phi_{f}\right]$ defines a homomorphism

$$
\operatorname{Homeo}_{B L P}\left(\mathbf{H}_{\infty}^{G}\right) \longrightarrow \operatorname{Vaut}(G) .
$$


It is time to prove the main theorem of this section.

Theorem 4.7. There is a canonical isomorphism

$$
\Gamma_{B L P}\left(\mathbf{H}_{\infty}^{G}\right) \stackrel{\cong}{\longrightarrow} \operatorname{Vaut}(G) .
$$

Proof. The kernel of the map

$$
\operatorname{Homeo}_{B L P}\left(\mathbf{H}_{\infty}^{G}\right) \longrightarrow \operatorname{Vaut}(G)
$$

contains $\mathrm{Homeo}_{0}\left(\mathbf{H}_{\infty}^{G}\right)$ since an isotopy of a baseleaf preserving self-homeomorphism necessarily fixes $\partial \mathbf{D}$. It follows that there is a natural homomorphism of $\Gamma_{B L P}\left(\mathbf{H}_{\infty}^{G}\right)$ into $\operatorname{Vaut}(G)$ which will be denoted $\rho$.

To prove that $\rho$ is injective, let $f$ be a baseleaf-preserving homeomorphism of $\mathbf{H}_{\infty}^{G}$. We may regard $f$ as a Teichmüller marking of $\mathbf{H}_{\infty}^{G}$. If the induced boundary action of $f$ on the baseleaf is trivial, then $f$ is Teichmüller equivalent to the identity homeomorphism. This implies that $f$ is isotopic to the identity.

To prove that $\rho$ is surjective, let $\phi: H \rightarrow K$ be a partial automorphism of $G$. We know $\phi$ is induced by an self-homeomorphism $f$ of $\partial \mathbf{D}$. It is possible to extend $f$ to an $H$-compatible self-homeomorphism of $\mathbf{D}$. (This is most directly accomplished by using the Douady-Earle extension operator [9].) It follows from Proposition 3.10 that there exists a self-homeomorphism of $\mathbf{H}_{\infty}^{G}$ that is mapped by $\rho$ to $[\phi]$.

We conclude this section with a pair of results concerning baseleaf preserving isometries of $G$-tagged solenoids.

Corollary 4.8. There is a canonical isomorphism

$$
\mathrm{Comm}_{\operatorname{Möb}(\mathbf{D})} G \stackrel{\cong}{\longrightarrow} \operatorname{Isom}_{B L P}^{+}\left(\mathbf{H}_{\infty}^{G}\right) .
$$

Proof. An injective map $i$ was established by Example 3.11. Since the baseleaf $\mathbf{D}$ is dense in $\mathbf{H}_{\infty}^{G}$, in order to prove surjectivity it is enough to show that if $f$ is an arbitrary orientation preserving, baseleaf preserving isometry of $\mathbf{H}_{\infty}^{G}$, then

$$
\left.f\right|_{\mathbf{D}} \in \operatorname{Comm}_{\mathrm{Möb}(\mathbf{D})} G .
$$

But this is true because $\left.f\right|_{\mathbf{D}}$ is a Möbius transformation and by Theorem $\left.4.6 f\right|_{\partial \mathbf{D}}$ is in the commensurator of $G$ in $\operatorname{Homeo}(\partial \mathbf{D})$.

Corollary 4.9. An orientation preserving, baseleaf preserving mapping class of $\mathbf{H}_{\infty}^{G}$ contains an isometry if and only if it induces a Möbius transformation on $\partial \mathbf{D}$.

Proof. Suppose $f$ is a homeomorphism which induces a Möbius transformation $\gamma$ on the boundary of the baseleaf. It follows that

$$
\gamma \in \operatorname{Comm}_{\operatorname{Möb}(\partial \mathbf{D})}(G) .
$$

Let $\widetilde{\gamma}$ be the unique Möbius transformation of $\mathbf{D}$ that agrees with $\gamma$ on $\partial \mathbf{D}$. By Example 3.11

$$
i(\widetilde{\gamma}) \in \operatorname{Isom}_{B L P}\left(\mathbf{H}_{\infty}^{G}\right)
$$

and $i(\widetilde{\gamma})^{-1} \circ f$ is a baseleaf preserving self-homeomorphism of $\mathbf{H}_{\infty}^{G}$ that induces the identity map on $\partial \mathbf{D}$. This implies $i(\widetilde{\gamma})$ is isotopic to $f$. The converse is trivial. 
4.3. The mapping class group of $\mathbf{X}_{\infty}$. We will now prove the topological version of Theorem 4.6. The key is to exploit the fact that the ideal boundary of the universal covering surface can be constructed in a purely topological fashion [11]. The section ends with an analysis of the topology of the full mapping class group of $\mathbf{X}_{\infty}$.

Theorem 4.10. There is a canonical isomorphism

$$
\Gamma_{B L P}\left(\mathbf{X}_{\infty}\right) \stackrel{\cong}{\longrightarrow} \operatorname{Vaut}\left(\pi_{1}(X, *)\right) .
$$

Proof. Choose a Fuchsian group $G$ that is isomorphic to $\pi_{1}(X)$ and choose a homeomorphism

$$
(X, *) \stackrel{h}{\longrightarrow} G \backslash \mathbf{D} .
$$

Let $x_{0}$ denote a choice of lift of $h(*)$ in $\mathbf{D}$. This choice induces an isomorphism of $\pi_{1}(X)$ onto $G$ as well as an isomorphism $h_{\#}$ of $\widehat{\pi_{1}}(X)$ onto $\widehat{G}$. The choice of $x_{0}$ also induces a homeomorphism $\widetilde{h}$ of $\widetilde{X}$ onto $\mathbf{D}$ and a corresponding homeomorphism of $\partial \widetilde{X}$ onto D. The assignment

$$
(t, x) \longmapsto\left(h_{\#}(t), \widetilde{h}(x)\right)
$$

induces a homeomorphism of $\mathbf{X}_{\infty}$ onto $\mathbf{H}_{\infty}^{G}$. We therefore obtain the commutative diagram

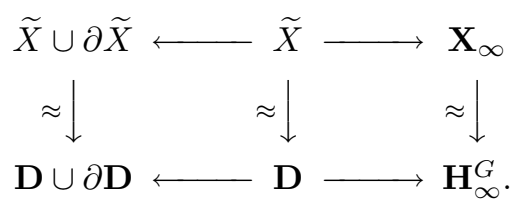

The theorem is proved by "pulling back" Theorem 4.7 via this diagram.

Theorem 4.10 may be regarded as a genus-independent version of the DehnNielsen-Baer theorem in the following manner. Let $(Y, *)$ be a finite covering surface of $(X, *)$. The basepoint preserving homeomorphism group of $Y$ is a subgroup of

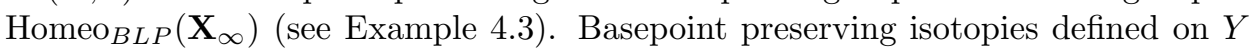
lift to baseleaf preserving isotopies at the solenoid level. It follows that $\Gamma(Y, *)$ is a subgroup of $\operatorname{Homeo}_{B L P}\left(\mathbf{X}_{\infty}\right)$; moreover, there is a canonical commutative diagram

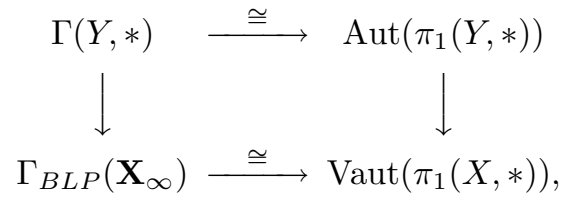

where the vertical homomorphisms are injective. By Theorem 2.15 the union (over all covering surfaces $Y)$ of the images of $\Gamma(Y, *)$ does not cover $\Gamma_{B L P}\left(\mathbf{X}_{\infty}\right)$. On the other hand it is not known if every baseleaf preserving mapping class of $\mathbf{X}_{\infty}$ can be decomposed into a finite number of elements each of which lies in some $\Gamma(Y, *)$.

A few comments on the role of basepoints are in order. There is an apparent asymmetry between the Dehn-Nielsen-Baer Theorem, a statement about basepoint preserving mapping class groups of surfaces, and Theorem 4.10, a statement about baseleaf preserving mapping classes of $\mathbf{X}_{\infty}$. We will now describe how this apparent asymmetry can be resolved in either direction.

Let $\mathrm{Homeo}_{*}\left(\mathbf{X}_{\infty}\right)$ denote the group of self-homeomorphisms that fix the canonical basepoint. The pointed mapping class group of $\mathbf{X}_{\infty}$, denoted $\Gamma\left(\mathbf{X}_{\infty}, *\right)$, is 
defined to be the quotient of $\mathrm{Homeo}_{*}\left(\mathbf{X}_{\infty}\right)$ by the subgroup of basepoint preserving self-homeomorphisms that are isotopically trivial (relative to the basepoint). It turns out that $\Gamma\left(\mathbf{X}_{\infty}, *\right)$ is nothing more than $\Gamma_{B L P}\left(\mathbf{X}_{\infty}\right)$. The proof requires the following fact.

Lemma 4.11. The group $\mathrm{Homeo}_{0}\left(\mathbf{X}_{\infty}\right)$ acts transitively on $\widetilde{X}$.

Proof. Let $p$ and $q$ be arbitrary points in $\widetilde{X}$. Choose a simple path $\alpha$ from $p$ to $q$ and a finite index subgroup $K$ in $\pi_{1}(X)$ that has a fundamental domain $D_{K}$ enclosing $\alpha$. Define a self-homeomorphism of $D_{K}$ by "dragging" $p$ to $q$ while leaving the boundary of $D_{K}$ fixed. Extend this map to a $K$-invariant self-homeomorphism $f$ of $\widetilde{X}$ that fixes $\partial X$. It is easy to extend $f$ to an isotopically trivial self-homeomorphism of $\mathbf{X}_{\infty}$ that maps $p$ to $q$.

Theorem 4.12. There is a canonical isomorphism

$$
\Gamma_{B L P}\left(\mathbf{X}_{\infty}\right) \stackrel{\cong}{\longrightarrow} \Gamma\left(\mathbf{X}_{\infty}, *\right) .
$$

Proof. By the previous lemma the group $\operatorname{Homeo}_{B L P}\left(\mathbf{X}_{\infty}\right)$ is generated by the subgroups $\mathrm{Homeo}_{0}\left(\mathbf{X}_{\infty}\right)$ and $\mathrm{Homeo}_{*}\left(\mathbf{X}_{\infty}\right)$. It follows that the map in question exists and is surjective. To prove injectivity it remains to verify that if an isotopically trivial self-homeomorphism of $\mathbf{X}_{\infty}$ preserves the canonical basepoint, then it is isotopically trivial relative to the basepoint. The proof of this fact resembles the proof of the previous lemma.

In this way the "missing" basepoint can be restored to Theorem 4.10.

Another way to reconcile the apparent asymmetry is to view the basepoints as being "absorbed at infinity". The lifts of the basepoints of all the surfaces in the canonical covering tower of $(X, *)$ form a "lattice" $\mathcal{L}$ in $\widetilde{X}$ that accumulates at every point in $\partial \widetilde{X}$. Consider a basepoint preserving homeomorphism of a covering surface $(Y, *)$. Such a map preserves a finite index sublattice of $\mathcal{L}$ and therefore determines the induced action on $\partial \widetilde{X}$. Moreover, a basepoint preserving isotopy of this map induces an isotopy of $\widetilde{X}$ that fixes $\partial \widetilde{X}$. With this view it is evident that basepoints provide a convenient means by which to control the ideal boundary of the baseleaf, and it is the ideal boundary that is fundamental to understanding $\Gamma_{B L P}\left(\mathbf{X}_{\infty}\right)$.

It should be noted that the unpointed mapping class group of $Y$, which is isomorphic to

$$
\operatorname{Aut}\left(\pi_{1}(Y)\right) / \operatorname{Inn}\left(\pi_{1}(Y)\right)
$$

does not appear naturally as a subgroup of $\operatorname{Vaut}\left(\pi_{1}(X)\right)$ since inner automorphisms of $\pi_{1}(X)$ induce isotopically nontrivial homeomorphisms of $\mathbf{X}_{\infty}$.

Now consider $\Gamma\left(\mathbf{X}_{\infty}\right)$, the full mapping class group of $\mathbf{X}_{\infty}$. Since it is defined as the quotient of topological groups, it inherits a quotient topology. The first step in analyzing this topology is to notice that the virtual automorphism group of $\pi_{1}(X)$ is topologized by the inclusion

$$
\operatorname{Vaut}\left(\pi_{1}(X)\right) \longrightarrow \operatorname{Homeo}(\partial \tilde{X}) .
$$

Given $\gamma$ in $\pi_{1}(X)$, let $i_{\gamma}$ denote the corresponding inner automorphism of $\pi_{1}(X)$. The map $\gamma \mapsto i_{\gamma}$ induces an inclusion of $\pi_{1}(X)$ into $\operatorname{Vaut}\left(\pi_{1}(X)\right)$. Define an action of $\pi_{1}(X)$ on the product space

$$
\widehat{\pi_{1}}(X) \times \operatorname{Vaut}\left(\pi_{1}(X)\right)
$$


by setting

$$
\gamma \cdot(t, \phi)=\left(t \gamma^{-1}, i_{\gamma} \circ \phi\right)
$$

Theorem 4.13. There is a canonical homeomorphism

$$
\Gamma\left(\mathbf{X}_{\infty}\right) \stackrel{\approx}{\longrightarrow} \widehat{\pi_{1}}(X) \times_{\pi_{1}(X)} \operatorname{Vaut}\left(\pi_{1}(X)\right) .
$$

Proof. By Theorem $4.4 \operatorname{Homeo}\left(\mathbf{X}_{\infty}\right)$ is homeomorphic to

$$
\widehat{\pi_{1}}(X) \times_{\pi_{1}(X)} \text { Homeo }_{B L P}\left(\mathbf{X}_{\infty}\right) .
$$

The canonical surjective homomorphism of $\operatorname{Homeo}_{B L P}\left(\mathbf{X}_{\infty}\right)$ onto $\operatorname{Vaut}\left(\pi_{1}(X)\right)$, which will be denoted $\rho$, is clearly continuous. Notice that $\rho$ maps a covering translation $C_{\gamma}$ to the inner automorphism $i_{\gamma}$. It is easily verified that the assignment $\left(T_{s}, f\right) \mapsto(s, \rho(f))$ induces a continuous map

$$
\widehat{\pi_{1}}(X) \times_{\pi_{1}(X)} \operatorname{Homeo}_{B L P}\left(\mathbf{X}_{\infty}\right) \stackrel{\varrho}{\longrightarrow} \widehat{\pi_{1}}(X) \times_{\pi_{1}(X)} \operatorname{Vaut}\left(\pi_{1}(X)\right) .
$$

Suppose that $T_{s} \circ f$ is isotopic to $T_{u} \circ g$. These homeomorphisms map the baseleaf to the same leaf; therefore, there exists $\gamma$ in $\pi_{1}(X)$ such that $s=u \gamma$. It follows that

$$
T_{s} \circ f=\left(T_{u \gamma}\right) \circ f=T_{u} \circ\left(C_{\gamma} f\right) .
$$

Consequently, $C_{\gamma} \circ f$ is isotopic to $g$ and

$$
\rho(g)=\rho\left(C_{\gamma} \circ f\right)=i_{\gamma} \circ \rho(g) .
$$

We conclude that $\varrho\left(T_{s} \circ f\right)=\varrho\left(T_{u} \circ g\right)$. The map $\varrho$ is therefore defined on $\Gamma\left(\mathbf{X}_{\infty}\right)$ and it is not hard to show that $\varrho$ is a homeomorphism.

Remark. The preceding theorem is a statement about the topology of $\Gamma\left(\mathbf{X}_{\infty}\right)$ and does not shed light on its group structure.

Corollary 4.14. There is a canonical homeomorphism

$$
\operatorname{Isom}^{+}\left(\mathbf{H}_{\infty}^{G}\right) \stackrel{\approx}{\longrightarrow} \widehat{G} \times{ }_{G} \operatorname{Comm}_{\mathrm{Möb}(\mathbf{D})} G .
$$

4.4. The unit tangent bundle of $\mathbf{H}_{\infty}^{G}$. Let $U T(\mathbf{D})$ denote the unit tangent bundle of D. By choosing an element $\zeta$ in $U T(\mathbf{D})$ and identifying $\zeta$ with the identity transformation in $\operatorname{Möb}(\mathbf{D})$, the formula $\gamma \mapsto \gamma \cdot \zeta$ defines a homeomorphism of Möb(D) onto $U T(\mathbf{D})$. If $G$ is a Fuchsian group, then $U T(G \backslash \mathbf{D})$ is homeomorphic to $G \backslash \operatorname{Möb}(\mathbf{D})$.

The unit tangent bundle $U T\left(\mathbf{H}_{\infty}^{G}\right)$ is constructed similarly. There is a natural projection

$$
\widehat{G} \times U T(\mathbf{D}) \longrightarrow U T\left(\mathbf{H}_{\infty}^{G}\right)
$$

obtained by taking the quotient of $\widehat{G} \times U T(\mathbf{D})$ by the natural action of $G$. Choose a unit vector $\zeta$ tangent to the baseleaf in $\mathbf{H}_{\infty}^{G}$ and identify $\zeta$ with the identity Möbius transformation. One thereby obtains a homeomorphism

$$
\widehat{G} \times \operatorname{Möb}(\mathbf{D}) \stackrel{\approx}{\longrightarrow} \widehat{G} \times U T(\mathbf{D}) .
$$

Since the map is $G$-equivariant it induces a homeomorphism

$$
\widehat{G} \times_{G} \operatorname{Möb}(\mathbf{D}) \stackrel{\approx}{\longrightarrow} U T\left(\mathbf{H}_{\infty}^{G}\right) .
$$

Here is a canonical model for $U T(\mathbf{D})$. Let $Y$ be the space of distinct triples of points in $\partial \mathbf{D}$ taken in counter-clockwise order. Each point in $Y$ determines an element of $U T(\mathbf{D})$ as indicated in Figure 7. If $G$ is a Fuchsian group, then it acts 


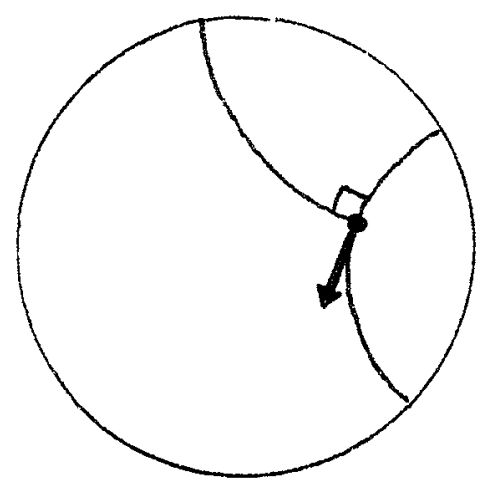

FiguRE 7. Determining a point in $U T(\mathbf{D})$.

on $Y$ in the obvious manner; the quotient $G \backslash Y$ is canonically homeomorphic to $U T(G \backslash \mathbf{D})$.

It is known that the mapping class group of a surface does not act smoothly on the surface [16] - in other words, there is no faithful map

$$
\Gamma(G \backslash \mathbf{D}) \longrightarrow \operatorname{Diffeo}(G \backslash \mathbf{D}) \text {. }
$$

It is not known if $\Gamma(G \backslash \mathbf{D})$ acts faithfully on $G \backslash \mathbf{D}$ by homeomorphisms; however, Cheeger and Gromov used the space $Y$ to exhibit a faithful, natural action

$$
\Gamma(G \backslash \mathbf{D}) \longrightarrow \text { Homeo }(U T(G \backslash \mathbf{D}))
$$

(see [8], p. 54). The spirit of the construction is:

(1) Lift a mapping class to $\mathbf{D}$ and let it act on $\partial \mathbf{D}$.

(2) Use the induced action on $Y$ to define a homeomorphism of $U T(\mathbf{D})$.

(3) Check that this procedure is suitably equivariant.

A generalization of this procedure can be used to construct a faithful action of $\Gamma_{B L P}\left(\mathbf{H}_{\infty}^{G}\right)$ on $U T\left(\mathbf{H}_{\infty}^{G}\right)$. First observe that there is a canonical homeomorphism

$$
U T\left(\mathbf{H}_{\infty}^{G}\right) \stackrel{\simeq}{\longrightarrow} \widehat{G} \times_{G} Y .
$$

Since $\operatorname{Vaut}(G)$ acts naturally on both $\partial \mathbf{D}$ and $\widehat{G}$, it follows that $\operatorname{Vaut}(G)$ acts naturally and equivariantly on $\widehat{G} \times Y$. Consequently there is a natural, injective homomorphism

$$
\Gamma_{B L P}\left(\mathbf{H}_{\infty}^{G}\right) \longrightarrow \operatorname{Homeo}\left(U T\left(\mathbf{H}_{\infty}^{G}\right)\right) .
$$

4.5. The universal euclidean solenoid. The methods contained in this article can be modified to analyze $\mathbf{E}_{\infty}$, the universal euclidean solenoid. To simplify arguments we will concentrate on the "standard" marked solenoid by taking $X=$ $\mathbf{R}^{2} / \mathbf{Z}^{2}$. In this case there is a canonical model

$$
\mathbf{E}_{\infty} \approx \widehat{\mathbf{Z}}^{2} \times \mathbf{Z}^{2} \mathbf{R}^{2}
$$

with marked baseleaf $\widetilde{X} \approx \mathbf{R}^{2}$. Two geodesics in $\mathbf{R}^{2}$ are declared to be equivalent if they are parallel. Topologized in the obvious fashion this set of equivalence classes becomes an "ideal boundary" $\partial \widetilde{X}$ which is homeomorphic to $\mathbf{P}^{1} \mathbf{R}$. The uniform structure on $\mathbf{E}_{\infty}$ can be exploited to prove the existence of an action

$$
\Gamma_{B L P}\left(\mathbf{E}_{\infty}\right) \longrightarrow \operatorname{Homeo}(\partial \widetilde{X}) .
$$


The image of this map can be recognized as $P G L(2, \mathbf{Q})$. Unlike the hyperbolic case, this map has a kernel. This kernel is isomorphic to $\mathbf{Q}^{*}$ and corresponds to rational hometheties of the marked baseleaf. Such maps are isotopically nontrivial but act trivially on the ideal boundary. The upshot is that

$$
\Gamma_{B L P}\left(\mathbf{E}_{\infty}\right) \cong G L(2, \mathbf{Q}) \cong \operatorname{Vaut}\left(\pi_{1}(X)\right) .
$$

As in the hyperbolic case the full mapping class group of $\mathbf{E}_{\infty}$ is homeomorphic to

$$
\widehat{\mathbf{Z}}^{2} \times \mathbf{Z}^{2} G L(2, \mathbf{Q}) .
$$

In the euclidean case, however, it is possible to discern the group structure. On one hand translations of the marked baseleaf - in particular homeomorphisms induced by elements of $\pi_{1}(X)=\mathbf{Z}^{2}$ - are isotopically trivial. It follows that $\mathbf{Z}^{2}$ acts trivially on the right-hand factor. On the other hand, since $\mathbf{Z}$ is abelian the quotient space $\widehat{\mathbf{Z}}^{2} / \mathbf{Z}^{2}$ is in fact a group -indeed, a subgroup of $\operatorname{Homeo}\left(\mathbf{E}_{\infty}\right)$. Via the action

$$
G L(2, \mathbf{Q}) \longrightarrow \operatorname{Sym}\left(\widehat{\mathbf{Z}}^{2} / \mathbf{Z}^{2}\right)
$$

(see Section 2.4) construct the semidirect product

$$
\Gamma=\widehat{\mathbf{Z}}^{2} / \mathbf{Z}^{2} \ltimes G L(2, \mathbf{Q}) .
$$

There is a natural injective homomorphism

$$
\Gamma \longrightarrow \operatorname{Homeo}\left(\mathbf{E}_{\infty}\right) \text {. }
$$

It can be verified that $\Gamma$ is canonically isomorphic to the full mapping class group $\Gamma\left(\mathbf{E}_{\infty}\right)$ and this homomorphism is a splitting of the surjection

$$
\operatorname{Homeo}\left(\mathbf{E}_{\infty}\right) \longrightarrow \Gamma\left(\mathbf{E}_{\infty}\right) \text {. }
$$

\section{REFERENCES}

1. R. Baer, Isotopien von Kurven auf orientierbaren, geschlossenen Flächen und ihr Zusammenhang mit der topologischen Deformation der Flächen, J. Reine Angew. Math. 159 (1928), 101-116.

2. I. Biswas, M. Mitra, and S. Nag, Thurston boundary of the Teichmüller spaces and the commensurability modular group, Conform. Geom. Dyn. 3 (1999), 50-66. MR 2000b:32030

3. I. Biswas and S. Nag, Commensurability automorphism groups and infinite constructions in Teichmüller theory, C. R. Acad. Sci. Paris Ser. I Math. 327 (1998), no. 1, 35-40. MR 99i:32026

4. I. Biswas and S. Nag, Jacobians of Riemann surfaces and the Sobolev space $H^{1 / 2}$ on the circle, Math. Res. Lett. 5 (1998), no. 3, 281-292. MR 99g:58009

5. I. Biswas and S. Nag, Weil-Petersson geometry and determinant bundles on inductive limits of moduli spaces, Lipa's legacy (New York, 1995), 51-80, Contemp. Math., 211, Amer. Math. Soc., Providence, RI, 1997. MR 99m:14050

6. I. Biswas and S. Nag, Limit constructions over Riemann surfaces and their paramater spaces, and the commensurability group actions, Selecta Math. (N.S.) 6 (2000), no. 2, 185-224. MR 2002f: 32026

7. I. Biswas, S. Nag, and D. Sullivan, Determinant bundles, Quillen metrics and Mumford isomorphisms over the universal commensurability Teichmüller space, Acta Math. 176 (1996), 145-169. MR 97h:32030

8. A. J. Casson and S. A. Bleiler, Automorphisms of surfaces after Nielsen and Thurston, London Math. Soc. Student Texts, vol. 9, Cambridge Univ. Press, Cambridge and New York, 1988. MR 89k:57025

9. A. Douady and C. J. Earle, Conformally natural extension of homeomorphisms of the circle, Acta Math. 157 (1986), no. 1-2, 23-48. MR 87j:30041

10. D. B. A. Epstein, Curves on 2-manifolds and isotopies, Acta Math. 115 (1966), 83-107. MR 35:4938 
11. W. Floyd, Group completions and limit sets of Kleinian groups, Invent. Math. 57 (1980), no. 3, 205-218. MR 81e:57002

12. R. H. Fox, On Fenchel's conjecture about F-groups, Mat. Tidsskr. B (1952), 61-65. MR 14:843c

13. A. G. Kurosh, Theory of Groups, Vol. II, Chelsea, New York, 1956. MR 18:188f

14. A. I. Mal'cev, Nilpotent torsion-free groups, Izvestiya Akad. Nauk SSSR. 13 (1949), 201-212. MR 10:507e

15. F. Menegazzo and M. J. Tomkinson, Groups with trivial virtual automorphism group, Israel J. Math. 71 (1990), no. 3, 297-308. MR 92c:20072

16. S. Morita, Characteristic classes of surface bundles, Bull. AMS 11 (1984), no. 2, 386-388. MR 85j:55032

17. D. Mumford, Tata lectures on theta. I (with C. Musili, M. Nori, E. Previato and M. Stillman), Progress in Mathematics, vol. 28, Birkhäuser Boston, Inc., Boston, MA, 1983. MR 85h:14026

18. S. Nag, Mathematics in and out of string theory, Topology and Teichm-ller spaces (Katinkulta, 1995), 187-220, World Sci. Publishing, River Edge, NJ, 1996. MR 99j:58221

19. J. Nielsen, Untersuchungen zur Topologie der geschlossenen zweiseitigen Flächen, Acta. Math. 50 (1927), 189-358.

20. C. Odden, The virtual automorphism group of the fundamental group of a closed surface, Ph.D. thesis, Duke University, 1997.

21. D. Sullivan, Linking the universalities of Milnor-Thurston, Feigenbaum and Ahlfors-Bers, Topological methods in modern mathematics (Stony Brook, NY, 1991), Publish or Perish, Houston, TX, 1993, pp. 543-564. MR 94c:58060

22. R. J. Zimmer, Ergodic theory and semisimple groups, Monographs in Mathematics, vol. 81, Birkhäuser Verlag, Basel and Boston, 1984. MR 86j:22014

Department of Mathematics and Computer Science, Amherst College, Amherst, MasSACHUSETTS 01002

Current address: Department of Mathematics, Phillips Academy, Andover, Massachusetts 01810

E-mail address: ctodden@andover.edu 